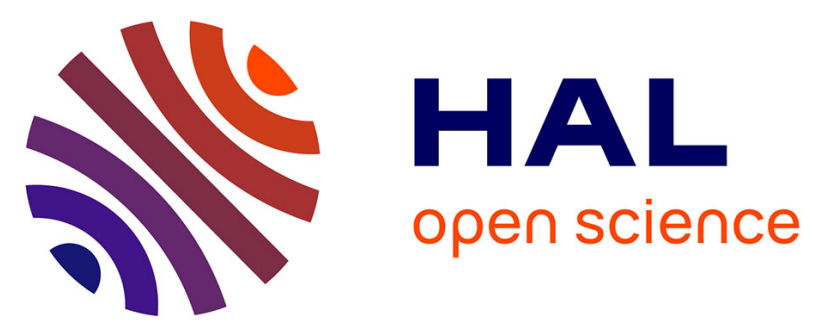

\title{
L'impact des stimuli informationnels d'un nouveau produit alimentaire sur les réactions affectives et cognitives du consommateur
}

Gaëlle Pantin-Sohier, Caroline Lancelot Miltgen

\section{- To cite this version:}

Gaëlle Pantin-Sohier, Caroline Lancelot Miltgen. L'impact des stimuli informationnels d'un nouveau produit alimentaire sur les réactions affectives et cognitives du consommateur. Recherche et Applications en Marketing (French Edition), 2012, 27 (1), pp.3-32. hal-01117036

HAL Id: hal-01117036

https://hal-audencia.archives-ouvertes.fr/hal-01117036

Submitted on 16 Feb 2015

HAL is a multi-disciplinary open access archive for the deposit and dissemination of scientific research documents, whether they are published or not. The documents may come from teaching and research institutions in France or abroad, or from public or private research centers.
L'archive ouverte pluridisciplinaire $\mathbf{H A L}$, est destinée au dépôt et à la diffusion de documents scientifiques de niveau recherche, publiés ou non, émanant des établissements d'enseignement et de recherche français ou étrangers, des laboratoires publics ou privés. 


\title{
L'IMPACT DES STIMULI INFORMATIONNELS D'UN NOUVEAU PRODUIT ALIMENTAIRE SUR LES REACTIONS AFFECTIVES ET COGNITIVES DU CONSOMMATEUR
}

\author{
GAËLLE PANTIN-SOHIER \\ MAITRE DE CONFERENCES, UNIVERSITE D’ ANGERS
}

Coordonnées professionnelles :

LUNAM Université

Université d'Angers

GRANEM (Groupe de Recherche ANgevin en Economie et Management)

UFR de Droit, d'Economie et de Gestion

13, Allée François Mitterrand

BP 13633

49036 Angers Cedex 01

TEL : 0241962155

Email : gaelle.pantin-sohier@univ-angers.fr

Coordonnées personnelles:

16 Allée Paul Signac

49240 Avrillé

TEL : 0661236544

Email : gaelle.pantin-sohier@wanadoo.fr

\author{
CAROline LANCElot Miltgen \\ MAITRE DE CONFERENCES, UNIVERSITE D’ ANGERS
}

Coordonnées professionnelles :

LUNAM Université

Université d'Angers

GRANEM (Groupe de Recherche ANgevin en Economie et Management)

UFR de Droit, d'Economie et de Gestion

13, Allée François Mitterrand

BP 13633

TEL : 0241226600

Email : caroline.miltgen@ univ-angers.fr

Coordonnées personnelles:

83, Boulevard Eugene Chaumin

49000 Angers

TEL : 0665012704

Email : caroline.miltgen@orange.fr 


\title{
L'impact des stimuli informationnels d'un nouveau produit alimentaire sur les réactions affectives et cognitives du consommateur
}

Résumé

L'objectif de cet article est de montrer l'influence simultanée de l'innovation produit (complémentarité de l'arôme) et de l'innovation packaging (design du produit à travers la prototypicalité du contenant et la quantité d'informations sur l'étiquette) lors du lancement d'une nouvelle boisson alcoolisée aromatisée. Ces éléments sont testés par le biais d'une expérimentation invitant 383 individus à évaluer la pertinence du mélange proposé, la nouveauté perçue de la boisson et leurs inférences en matière de goût (caractère sucré, douceur et intensité). Nous examinons en outre le rôle médiateur de ces variables sur l'agrément et celui de l'agrément sur l'intention de goûter le nouveau produit alimentaire. Les résultats confirment que la complémentarité de l'arôme influence positivement la pertinence perçue du mélange et négativement sa nouveauté perçue. L'analyse permet également d'identifier une relation linaire entre pertinence perçue et agrément alors que la relation entre nouveauté perçue et agrément suit une tendance quadratique (courbe en U).

Mots clés: Information visuelle, packaging, nouveauté, pertinence, agrément, goût, inférence.

\section{The emotional and cognitive effects of a new food product's informational stimuli on the consumer}

\begin{abstract}
The purpose of this article is to examine the influence of a product's innovation (complementarity of the flavour associated with the product) and packaging innovation (the type and amount of information on the packaging) when launching a new flavoured alcoholic beverage. These variables were tested through an experiment which consisted of inviting 383 respondents to judge the suitability of the mixture and the novelty of the drink and specify their opinions regarding taste (the sweetness and its intensity). Furthermore we examined the mediating role of the perceptions on the product agreement and that of the agreement on the subject's intention to taste the product. The results confirm that the fit of the flavour influences, in a positive way, the suitability perceived and in a negative way, the perceived novelty of the mixture. Also, the analysis shows that the relationship between perceived suitability and agreement is linear and the relationship between perceived novelty and agreement tends towards a second degree model.
\end{abstract}

Key words: Visual stimulus, packaging, novelty, suitability, pleasantness, taste, inference. 


\section{INTRODUCTION}

Lorsque les consommateurs choisissent un produit alimentaire, leur perception n'est pas seulement affectée par les caractéristiques sensorielles de celui-ci mais aussi par des idées générées par un ensemble de variables extrinsèques telles que le nom de marque, les photographies ou le packaging (Shifferstein, 2001). Cependant, peu de travaux ont examiné l'influence de ces variables extrinsèques sur la qualité perçue du produit (Deliza et Mc Fie, 1996). Les recherches ayant exploré ces relations (e.g. Tuorila et alii.1998) montrent que le packaging d'un aliment ou d'une boisson peut fournir un signal qui influencera les évaluations gustatives. La forme, la taille ou la couleur des produits ou de leur packaging sont en mesure d'influencer la qualité perçue des produits en jouant le rôle de réducteur d'incertitude (Sirieix, 1999). Afin de juger de la qualité d'un produit alimentaire, les consommateurs utilisent aussi des attributs de qualité intrinsèques tels que la flaveur. Néanmoins, force est de constater que les études mettant en évidence le lien entre les attributs intrinsèques du produit et les évaluations subjectives du produit demeurent limitées. Selon d'Hauteville (2003), l'un des enjeux de la recherche sensorielle est de mieux comprendre le lien entre les caractéristiques intrinsèques, contrôlables par l'industriel et la qualité perçue par les consommateurs. Cet enjeu devient primordial dans le contexte des nouveaux produits alimentaires où l'objectif des producteurs est de faire accepter l'innovation. L'innovation alimentaire concerne à la fois le packaging (sa forme, sa couleur, le matériau ...) et le produit lui-même (nouveaux goûts, nouvelles textures, multitexture, nouveaux ingrédients, etc).

La particularité de notre recherche est qu'elle inclut cette double dimension de l'innovation alimentaire dans le cadre du lancement d'une nouvelle boisson (cidre) alcoolisée aromatisée. Si l'innovation packaging s'appuie sur les études portant sur l'influence des composantes visuelles, l'innovation produit, pose inévitablement la question de l'acceptabilité des nouvelles associations alimentaires proposées aux consommateurs et fait référence aux fondements théoriques portant sur la catégorisation et la congruence. Il convient dès lors de mesurer la pertinence et la nouveauté perçue du produit. Radford et Bloch (2011) soulignent que la nouveauté d'un produit est largement communiquée à travers son design, l'innovation packaging pouvant renforcer la nouveauté perçue du mélange en interaction avec l'innovation produit. L'objectif de notre étude est double. Tout d'abord, elle vise à montrer comment le packaging (prototypicalité du contenant et présence versus absence sur l'étiquette de l'image de l'arôme) et la complémentarité du mélange boisson (cidre)-arôme influencent d'une part la 
perception de la pertinence du mélange proposé et la nouveauté perçue du produit et d'autre part génèrent des inférences en terme de goût et d'intensité de l'arôme. Cette recherche vise à identifier les sources d'informations (effets simples et d'interaction) utilisées par le consommateur pour générer des inférences (Mitchell et Olson, 1981 ; Graeff et Olson, 1994) sur un nouveau produit alimentaire, en l'occurrence ici un nouveau cidre aromatisé. Le second objectif de cette étude est de mettre en exergue les liens existant entre la nouveauté perçue d'un produit, la pertinence, les inférences gustatives, l'agrément et l'intention de goûter le nouveau produit proposé. Dans cette optique, nous présenterons dans un premier temps une revue de littérature portant sur les deux volets de l'innovation dans le secteur alimentaire en traitant successivement de l'innovation au niveau du produit alimentaire (acceptabilité, catégorisation et congruence) puis au niveau du packaging en mettant en avant son potentiel en tant que vecteur d'inférences. Ensuite, nous présenterons le modèle testé, décrirons la méthodologie employée et commenterons les résultats obtenus. Puis, nous conclurons par une dernière partie consacrée aux apports et limites de la recherche où nous discuterons les résultats et proposerons les voies de recherche à privilégier.

\section{L'INNOVATION DANS LE SECTEUR ALIMENTAIRE : UNE DOUBLE PERSPECTIVE}

\section{L'innovation alimentaire au niveau du produit : catégorisation, congruence et acceptabilité}

La problématique de l'innovation alimentaire se complexifie lorsqu'elle vise le produit luimême, c'est-à-dire sa présentation visuelle mais également sa composition (les saveurs associées, l'introduction d'un nouvel ingrédient ou une combinaison insolite). Si la couleur bleue est la couleur préférée des consommateurs à l'échelle internationale et qu'elle est communément admise sur les packagings dans l'univers alimentaire (ex: yaourts Ba de Nestlé ou biscuits Oreo de Nabisco), force est de constater qu'elle est très peu tolérée lorsqu'elle concerne le produit ingéré (Tysoe, 1985). De nombreux auteurs soulignent la complexité de l'acte alimentaire (Levi-Strauss, 1964) et la difficulté à identifier les origines des préférences (Zajonc et Markus, 1982). Les explications résident dans le paradoxe de l'omnivore (Fischler, 1999 ; Rozin, 1976). Selon Chiva (1992), l'état d'omnivore est à la fois, et contradictoirement, source de liberté et d'adaptation possible et source d'inquiétude face à l'inconnu, qui dérive de l'obligation de variété. L'auteur souligne que «le paradoxe réside dans ce tiraillement, dans cette contradiction, qui d'une part, permet l'innovation, la découverte mais d'autre part justifie la méfiance, le traditionalisme » ( $p$ 435). Dès lors, les 
mécanismes conduisant à l'acceptabilité alimentaire reposent majoritairement sur les principes de la catégorisation et la recherche de congruence.

La catégorisation conduit les consommateurs à traiter et évaluer toute expérience en fonction de la catégorie cognitive à laquelle elle fait référence (Alba et Hutchinson, 1987 ; Cohen et Basu, 1987 ; Loken et Ward, 1990). Plus précisément, les individus vont insérer les produits dans des catégories cognitives en fonction de leur degré de typicalité (Rosch et Mervis, 1975 ; Ladwein, 1995) ou de prototypicalité (Reed, 1972 ; Rosch, 1973 ; Veryzer, 1993 ; Veryzer et Hutchinson, 1998). Le jugement de typicalité est donc un mode d'évaluation holistique, qui permet de faciliter l'accessibilité aux nouvelles informations et leur utilisation ultérieure par l'individu (Amine et Glerant-Glikson, 2010). Il permet de traiter les stimuli nouveaux et de les rattacher à une ou plusieurs catégories cognitives qui structurent les connaissances de l'individu. Les recherches empiriques (e.g. Nedungadi et Hutchinson, 1985 ; Loken et Ward, 1990 ; Ladwein, 1998) menées sur la relation entre typicalité perçue et préférence des consommateurs conduisent aux même résultats, à savoir que la marque la plus typique remporte l'adhésion du consommateur, notamment en raison des heuristiques de choix simplificatrices utilisées (Desphande et Hoyer, 1983 ; Hoyer et Brown, 1990). En revanche, un produit innovant ne peut être associé à un schéma catégoriel donné (Meyers-Levy et Tybout, 1989) et peut générer une perception d'incongruence. Selon Lee (1995), le jugement de congruence est immédiat, il constitue la première impression sur le produit avant tout autre type de traitement. Il résulte à la fois d'une évaluation holistique et d'une évaluation plus analytique reposant sur les attributs saillants. Selon Maille (2007), le goût et la couleur devraient fortement influencer le jugement de congruence en matière de produit alimentaire. Notons que la congruence est multiforme. Il s'agit d'une correspondance structurelle entre deux entités (Mandler, 1982) qui comprend deux dimensions (Heckler et Childers, 1992) : d'une part, la pertinence, qui reflète dans quelle mesure l'information contenue dans le stimulus contribue à une identification claire du thème ou du message primaire communiqué, ou au contraire l'empêche ; d'autre part, le caractère attendu, qui fait référence au degré selon lequel un item ou une information tombe dans un schéma prédéterminé ou une structure évoquée par ce thème (Fleck-Dousteyssier et Korchia, 2006). Nous nous interrogeons ici sur les mécanismes de formation du jugement de congruence relatif à l'innovation alimentaire.

Dans le cadre de notre recherche sur un nouveau produit alimentaire, nous faisons l'hypothèse que la complémentarité de l'arôme avec la boisson proposée influencera à la fois la pertinence 
perçue et la nouveauté perçue de la combinaison. Concernant la pertinence, la plupart des travaux expliquent son existence sur la base d'une similarité perçue entre les deux entités, c'est-à-dire une ressemblance physique. Aaker et Keller (1990) montrent que la similarité physique n'est pas la seule forme de lien pouvant exister entre plusieurs entités et révèlent l'existence de trois autres sources de pertinence que sont la complémentarité, la transférabilité et la substituabilité. La complémentarité entre les deux entités résulte de l'utilité fonctionnelle d'une entité pour l'autre entité (par exemple le rasoir et la mousse à raser ; Maille \& Fleck, 2011). Martin \& Stewart (2001) parlent non pas de complémentarité mais de similarité d'usage pour des produits qui s'utilisent au même moment et dans un même objectif. Tel est le cas concernant le choix de l'arôme de notre boisson alcoolisée. Nous comparerons l'impact d'un arôme complémentaire à la boisson -correspondant à une expérience d'usage préalable- à celui d'un arôme qui n'est pas habituellement associé à la boisson choisie. Par ailleurs, le produit proposé lorsqu'il est doté d'un attribut non-conforme ou moins conforme au schéma de référence est perçu comme inattendu, c'est-à-dire plus nouveau (par exemple le fait qu'un jus de fruit naturel soit pétillant, Maille et Fleck, 2011). Shifferstein, Kole et Mojet (1999), dans leur travail sur les effets de la disconfirmation des attentes en matière alimentaire proposent l'hypothèse de curiosité selon laquelle les stimuli qui ne sont pas conformes aux attentes stimulent les individus du fait de leur caractère nouveau et intéressant. Dès lors, nous proposons de mesurer le degré de nouveauté perçue inféré par l'arôme intégré dans la boisson. Ainsi, la complémentarité de l'arôme avec la boisson devrait influencer le caractère attendu de la combinaison offerte et la nouveauté perçue. A l'instar de Christensen (1983), nous souhaitons également mesurer l'impact de la complémentarité de l'arôme sur la génération d'inférences gustatives et la perception d'intensité de l'arôme. Nous pouvons dès lors formuler l'hypothèse $\mathrm{H} 1$ suivante :

H1 : La complémentarité entre l'arôme et le produit auquel il est associé aura un effet direct et positif sur la pertinence perçue du mélange (H1a), direct et négatif sur la nouveauté perçue (H1b) puis direct et positif sur les inférences gustatives (doux H1c, sucré H1d) et l'intensité de l'arôme (H1e).

\section{Le packaging, vecteur d'inférences}

Lorsque le consommateur choisit un produit alimentaire (par exemple, une bouteille de vin, un yaourt ou un paquet de biscuits), sa décision d'achat repose fréquemment sur l'apparence visuelle des produits (Bloch, 1995 ; Fenko, Shifferstein \& Hekkert, 2010). Dans la majorité 
des cas, il n'est pas possible de goûter un produit alimentaire avant l'achat, la décision des acheteurs se prenant alors à partir de conditions non-sensorielles (Masson 2010). Parfois, le packaging conduit le consommateur à choisir le produit pour des raisons symboliques ou esthétiques (Creusen et Schoormans, 2005 ; Van Rompay, Pruyn et Tieke, 2009); il se procure ainsi l'édition limitée de la vodka de la marque Absolut pour des raisons principalement hédoniques. Par ailleurs, le packaging constitue régulièrement un vecteur d'identification, de reconnaissance et de distinction sur un marché de biens de consommation peu différenciés. Il est d'autant plus utile lorsque le consommateur manque d'informations sur le produit, par exemple lorsqu'il se retrouve face à un nouveau produit alimentaire. Dans ce cas précis, l'apparence visuelle du produit permet au consommateur de réaliser des inférences sur le produit, sur ses attributs et particulièrement sur son goût.

La forme du produit peut ainsi être considérée comme un déterminant majeur du choix des consommateurs et constituer un avantage compétitif vis-à-vis des concurrents (Bloch, 1995 ; Kotler et Rath, 1984 ; Oackley, 1990). Si elle est en mesure de véhiculer des messages spécifiques à la fois fonctionnels et symboliques (Hoegg et Alba, 2011), les recherches empiriques permettant d'isoler son influence sur le comportement du consommateur sont rares. Elles concernent principalement l'attention et la catégorisation (Berkowitz 1987 a et b ; Kreuzbauer et Malter, 2005 ; Schoormans et Robben, 1997), la prototypicalité (Veryzer, 1993, Veryzer et Hutchinson, 1998) ou les biais de perception visuelle (Krider, Raghubir et Krishna, 2001 ; Yang et Raghubir, 2006). Plusieurs auteurs ont analysé la question du lien entre l'angularité des formes et les perceptions de puissance. Ainsi, Berlyne (1976) montre que les formes anguleuses induisent des caractéristiques telles que l'énergie, la solidité et la force alors que les formes rondes ont tendance à susciter des comportements d'approche, une attitude amicale et une perception d'harmonie. Dans le contexte des produits alimentaires, Becker et al. (2011) analysent l'impact de la forme d'un packaging de yaourt sur les inférences gustatives et indiquent qu'un packaging anguleux conduit les consommateurs à attribuer un goût plus puissant au yaourt qu'un packaging arrondi.

Ces recherches montrent l'intérêt d'isoler les différentes caractéristiques du packaging afin de comprendre le rôle déterminant de chacune des variables étudiées, isolément et en interaction. Pour compléter ces recherches sur le packaging nous nous intéressons en premier lieu à l'impact de la prototypicalité du packaging sur les inférences réalisées par le répondant sur le plan gustatif. Nous faisons l'hypothèse que la prototypicalité du packaging est en mesure 
d'influencer à la fois l'intensité de l'arôme mais aussi les croyances en matière de goût pour deux critères (doux, sucré) ${ }^{1}$. Ainsi, nous pensons que le cidre aromatisé contenu dans le conditionnement le plus prototypique permettra au consommateur d'établir une concordance entre le produit et le schéma catégoriel auquel il est associé (Aurier et Fort, 2005) ; cette concordance perçue affecterait dès lors positivement le jugement du répondant. Le contenant fournit au consommateur une information qui peut réduire son incertitude s'il est prototypique de la catégorie évaluée.

Notre recherche étant consacrée à l'introduction d'une innovation alimentaire et à ses conditions d'acceptation, nous souhaitons aussi mesurer le degré de nouveauté perçue généré par l'apparence visuelle du produit. Comme le soulignent Radford et Bloch (2011), les chercheurs en innovation et les managers de produits gagneraient à connaître l'impact du design visuel des produits sur la communication de la nouveauté du produit. Alors que les recherches portant sur l'adoption des innovations sont nombreuses, elles se focalisent majoritairement sur la technologie du nouveau produit et ses caractéristiques plutôt que sur la manière dont la nouveauté est communiquée au consommateur. A l'instar de Radford et Bloch (2011), nous essayons de lier l'apparence visuelle du produit avec la nouveauté perçue et faisons l'hypothèse que la prototypicalité du contenant aura un impact négatif sur la nouveauté perçue alors que ce contenant sera jugé plus pertinent par les répondants. Le conditionnement le plus prototypique est pertinent car le lien associatif entre la boisson et ce type de conditionnement fait sens dans l'esprit des consommateurs, notamment avec les éléments présents en mémoire (Heckler et Childers, 1992). Sur la base de la revue de littérature exposée précédemment, nous pouvons dès lors formuler l'hypothèse $\mathrm{H} 2$ suivante : H2 : La boisson contenue dans le packaging le plus prototypique sera jugée plus pertinente (H2a), moins nouvelle (H2b), plus douce (H2c), plus sucrée (H2d) et plus intense (H2e).

En second lieu, nous avons pour objectif d'analyser l'impact de la quantité d'information présente sur le packaging. Underwood, Klein et Burke (2001) indiquent que la présence d'une image sur le packaging de marques plus ou moins connues de margarine, de bacon ou de friandises augmente significativement l'attention pour les marques peu connues. D'une manière générale, les consommateurs semblent préférer les packagings contenant une image

\footnotetext{
${ }^{1}$ Nous reprenons les évaluations gustatives les plus fréquemment utilisées dans les recherches portant sur les boissons aromatisées alcoolisées ou non (Pangborn, 1960 ; Pangborn \& Hansen, 1962, Johnson \& Clydesdale, 1982, Johnson \& al., 1982 ; Johnson, Dzendolet \& Clydesdale, 1983 ; Lavin et Lawless, 1998 ; Zellner et Kautz, 1990).
} 
et attribuent alors aux produits une meilleure évaluation si l'image du produit est attractive (Underwood et Klein, 2002). Enfin, la présence d'images est également en mesure de modifier la perception du volume des objets selon la complexité visuelle du produit (Garber, Hyatt et Boya, 2008). Nous suggérons alors que l'ajout d'une image (dans notre étude, celle de l'arôme) sur le packaging est en mesure de modifier la perception de la pertinence de l'association, la nouveauté perçue du mélange, l'intensité perçue de l'arôme et les inférences gustatives vis-à-vis du produit.

H3 : La présence de l'image de l'arôme choisi sur le packaging a un effet direct et positif sur la pertinence (H3a), la nouveauté (H3b), l'intensité de l'arôme (H3e), et les évaluations gustatives du produit (H3c: doux, H3d : sucré).

Un résumé des hypothèses formulées sur la base des recherches précédemment mentionnées est présenté en annexe 1. Nous prévoyons en outre l'existence d'effets d'interaction entre les trois variables indépendantes étudiées dans cette recherche. Nous cherchons à montrer que la communication d'un goût spécifique, d'un produit nouveau ou d'une combinaison pertinente peut être améliorée ou renforcée en agissant sur ces trois variables simultanément. Ainsi, nous faisons par exemple l'hypothèse qu'un produit associé à un arôme peu complémentaire et proposé dans un packaging peu prototypique sera perçu comme plus nouveau que celui combinant un arôme complémentaire et un packaging plus prototypique. Si les recherches antérieures permettent d'émettre des hypothèses spécifiques concernant les effets principaux, le caractère exploratoire de notre étude rend plus complexe la déduction d'hypothèses concernant les effets d'interaction. En revanche, nous testerons l'ensemble des effets d'interaction rendus possibles par le plan expérimental afin de montrer la puissance de l'impact des variables lorsqu'elles sont considérées simultanément.

Le rôle médiateur de la pertinence, de la nouveauté et des évaluations sensorielles sur l'agrément envers le produit

Notre recherche a également pour objectif de distinguer l'influence de la pertinence et de la nouveauté perçue sur l'agrément envers la boisson. La plupart des travaux valident le fait que pour être plus efficace (en termes de mémorisation de la publicité, d'évaluation du parrainage, de l'endossement ou de l'extension) que la congruence, l'incongruence doit être «non attendue » mais pertinente (Heckler et Childers, 1992 ; Lee et Mason, 1999; Masterson, 2005 ; Smith, Chen et Yang, 2008). Fleck et Maille (2010) soulignent que ces résultats ne concernent que la conception publicitaire et le parrainage et qu'il serait intéressant de vérifier 
leur stabilité dans d'autres domaines, ce que nous nous proposons de faire ici. Nous visons ainsi à mesurer l'impact de la pertinence et de la nouveauté perçue (caractère inattendu) sur l'agrément afin d'identifier le type d' «incongruence idéale ». En effet, l'évaluation distincte de la pertinence et de la nouveauté perçue, permettent dans notre recherche de comprendre les mécanismes de formation du jugement de congruence ou d'incongruence en fonction des combinaisons proposées au consommateur.

Tout d'abord, la plupart des travaux en comportement du consommateur indiquent que la congruence affecte positivement le jugement. Maille (2000) montre par exemple que les shampoings parfumés avec une odeur fortement congruente (versus faiblement) sont mieux perçus d'un point de vue hédonique, utilitaire et symbolique. Lichtlé (2002) indique que les produits testés sont mieux évalués lorsque la couleur et le contenu de l'annonce sont congruents. Christensen (1983) montre que la congruence des couleurs, mais aussi leur intensité, a un effet direct sur les seuils de perception des autres stimuli, et sur la préférence exprimée en termes d'odeur ou de goût. Le consommateur se contente de transférer l'attitude envers la catégorie cognitive du produit vers le produit à évaluer. Toutefois, la perception d'incongruence induit un traitement analytique de l'information qui affecterait négativement l'information (Lee, 1995 et Maille, 2007). Cette incongruence semble en revanche mieux acceptée et positivement évaluée lorsqu'elle concerne des innovations. Selon Campbell et Goodstein (2001), dans le cas du lancement d'un nouveau produit, l'effort cognitif supplémentaire que nécessite le traitement d'une information légèrement incongrue aboutirait à une plus grande satisfaction et une facilité d'acceptation de la nouveauté. Mandler (1982) explique alors la préférence des individus pour les produits modérément incongruents. Tout d'abord, ces derniers sont compréhensibles relativement facilement et appréhendés de manière holistique (Stayman, Alden et Smith, 1992). Ensuite, l'individu semble positivement influencé par le caractère stimulant de la nouveauté et par le fait d'avoir été capable de trouver une explication à l'incongruence perçue (Mandler, 1982). Maille (2007) montre, par exemple, que la perception d'une incongruence modérée relative à l'intensité des propriétés sensorielles d'un produit peut générer une attitude aussi favorable à l'égard du produit qu'une congruence totale (si l'incongruence modérée est générée par une intensité qui est perçue comme excessive par rapport aux attentes). L'objectif de cet article est d'isoler l'impact de la pertinence sur l'agrément envers le produit. Nous faisons l'hypothèse d'une relation linéaire entre la pertinence du mélange et l'agrément. Ainsi, nous serons en mesure d'indiquer si la 
non-pertinence rend véritablement l'incongruence trop forte et donc inefficace par opposition à l'information inattendue, qui elle, éveille l'attention et peut être perçue comme innovante et intéressante (Meyers-Levy et Tybout, 1989) si elle a du sens. La relation entre la pertinence et l'agrément doit logiquement suivre une relation linéaire, l'agrément évoluant positivement selon le degré de pertinence, telle que stipulée à travers l'hypothèse suivante :

H4 : Plus le mélange sera jugé pertinent par les consommateurs, plus l'agrément envers le produit sera favorable.

Selon Gielens \& Steenkamp (2007), la nouveauté perçue joue un rôle clé en tant que vecteur d'acceptation des nouveaux produits manufacturés dans les quatre pays étudiés (France, Allemagne, Espagne et Angleterre). Ils ajoutent que les analystes du secteur industriel indiquent que la croissance des ventes de produits manufacturés dépend de leur capacité à proposer des innovations et à les commercialiser avec succès. Nous vérifierons dans quelle mesure la nouveauté perçue de notre produit influence l'agrément pour ce produit et étudierons quelle est la nature de la relation entre ces deux variables. Steenkamp et Gielens (2003) montrent qu'il existe une relation en U entre la nouveauté perçue et la probabilité d'essai du produit. Cette relation peut s'expliquer par les deux facteurs sous-jacents de complexité et d'avantage relatif, qui augmentent tous les deux avec la nouveauté mais pas de manière linéaire. Plus un produit est nouveau, plus son avantage relatif vis-à-vis des produits existants est important (Gatignon, \& Xuereb, 1997), ce qui peut avoir un effet positif sur l'acceptation par le consommateur. D'un autre côté, plus le produit est nouveau, plus il est complexe, réduisant l'acceptation (Rogers, 1995). Si les produits modérément nouveaux ne sont pas meilleurs en termes d'avantage relatif que les nouveaux produits incrémentaux alors qu'ils ne sont pas beaucoup moins complexes que les produits réellement nouveaux, il en résulte une relation entre la nouveauté et l'acceptation suivant une courbe en $U$. Les innovations incrémentales et plus radicales seraient ainsi préférées par rapport aux produits dont le degré de nouveauté serait intermédiaire. Ces derniers génèrent en effet un niveau de complexité trop élevé par rapport aux innovations de nature incrémentale et un avantage relatif trop faible vis-à vis des innovations plus radicales ${ }^{2}$. Nous souhaitons vérifier l'existence de la relation en testant l'hypothèse suivante :

\footnotetext{
${ }^{2}$ Notons que Steenkamp et Gielens(2003) poursuivent en indiquant qu'un faible degré de nouveauté favorise une évaluation positive mais que l'attraction de l'innovation peut être à nouveau élevée à un niveau de forte nouveauté.
} 
H5 : Le lien entre nouveauté et agrément suit une courbe en $U$ : de faibles et forts degrés de nouveauté sont préférés à des degrés de nouveauté modérés.

De même, nous testons un effet direct des évaluations gustatives (doux, sucré, intensité) sur l'agrément envers le produit alimentaire. En revanche, le caractère exploratoire de notre étude ne nous permet pas de formuler d'hypothèses spécifiques quant au signe des relations.

H6 : Les évaluations gustatives ont un impact direct sur l'agrément envers le produit

Finalement, nous testerons également l'impact de l'agrément sur l'intention de goûter en proposant l'hypothèse $\mathrm{H} 7$ suivante :

H7 : L'agrément influence positivement l'intention de goûter le produit

Le modèle présenté présente les hypothèses qui seront testées dans le cadre de cette recherche. [Insérer Figure 1]

\section{METHODOLOGIE}

Le produit alimentaire que nous avons choisi d'étudier dans le cadre de cette recherche est un cidre aromatisé. Le choix du produit repose sur la forte tendance actuelle à l'aromatisation des boissons alcoolisées ou non (thés glacés, eaux minérales) et l'existence de ce type de boisson dans d'autres pays que la France (cidre néo-zélandais aromatisé à la poire de la marque Saxton par exemple). Nous souhaitons donc évaluer les réactions des consommateurs français face à cette nouvelle boisson aromatisée. Pour pouvoir tester l'effet de la complémentarité de l'arôme associé au produit/cidre et des caractéristiques du packaging proposé (tant en ce qui concerne la prototypicalité du contenant que la présence ou non de l'arôme sur l'étiquette) sur l'acceptabilité du produit, l'expérimentation s'est imposée comme la technique la plus appropriée. Une démarche expérimentale permet en effet de faire varier les trois facteurs susceptibles, d'après nos hypothèses, d'influencer l'acceptabilité alimentaire de cette nouvelle boisson aromatisée, tout en contrôlant l'effet potentiel de facteurs exogènes tels que le niveau d'implication du répondant vis-à-vis de la catégorie de produits/services étudiée, son genre et sa région d'origine ${ }^{3}$. Le contrôle de l'effet potentiel de ces éléments exogènes a été réalisé par une affectation aléatoire des individus interrogés aux différents traitements et par une introduction de ces variables dans nos analyses ultérieures.

\footnotetext{
${ }^{3}$ S'agissant de mesurer l'agrément envers un produit alimentaire, il est nécessaire de contrôler l'implication du répondant envers la catégorie de produits concernée. Le genre est la seule variable sociodémographique contrôlée, l'échantillon étant relativement homogène concernant l'âge, la CSP voire le niveau d'études. La région d'origine est une variable importante puisqu'il s'agit d'un produit fortement ancré sur le plan local.
} 


\section{La procédure expérimentale}

Le design de cette expérience repose sur un plan factoriel complet de type 2 x 2 x 2 (deux arômes, l'un complémentaire l'autre non ; deux niveaux -faible vs. élevée- de prototypicalité du packaging et deux niveaux -absence vs. présence- de l'image de l'arôme sur l'étiquette) en inter-sujets (chaque répondant n'a évalué qu'une seule possibilité sur les huit).

L'expérience a consisté tout d'abord à manipuler l'arôme associé à la boisson testée et en l'occurrence la complémentarité de l'arôme associé au cidre. Suite à une étude préalable où différents arômes associés au cidre étaient proposés afin de définir le niveau de complémentarité de chacun d'eux, deux arômes ont été retenus, l'un correspondant à un arôme complémentaire (le cassis), l'autre à un arôme peu complémentaire (le caramel). Ce résultat peut s'expliquer par le fait que le cassis est majoritairement utilisé par les consommateurs de cidre pour élaborer des kirs tandis que le caramel est totalement nouveau puisqu'il n'existe pas -à notre connaissance- de boisson alcoolisée associée à cette saveur. Les deux ingrédients associés étaient clairement identifiés sur l'étiquette par leur nom (cassis/caramel) et la couleur associée (violet et jaune-orangé) (cf. Annexe 2). La seconde manipulation a consisté à agir sur la prototypicalité du contenant en présentant la boisson soit dans une bouteille (conditionnement le plus prototypique), soit dans une canette (moins prototypique). La bouteille est le packaging le plus conforme aux codes visuels de la catégorie de produit cidre. La nouvelle boisson contenue dans la bouteille présente dès lors une ressemblance familiale forte (Loken \& Ward, 1990) avec les autres produits de sa catégorie et peut être considérée comme fortement typique de la catégorie de produit cidre. A l'inverse la présentation du cidre en canette constitue pour le répondant une distorsion prototypique et rend la catégorisation difficile. Pour des questions de contrôle, la contenance est identique dans les deux cas $(33 \mathrm{cl})$ ce qui correspond au format courant pour une boisson. Les répondants interrogés à ce sujet sont $89,7 \%$ à consommer habituellement le cidre en bouteille de $75 \mathrm{cl}$. La présentation du cidre sous un autre format -type de contenant (canette) et contenance $(33 \mathrm{cl})$ - est donc considérée ici comme innovante ${ }^{4}$. Enfin, la dernière manipulation concerne la présence ou non de l'image de l'arôme (baies de cassis ou morceaux de caramel) sur l'étiquette figurant sur le packaging de la boisson aromatisée.

\footnotetext{
${ }^{4}$ Le produit présenté crée de nouvelles conditions d'usage (consommation individuelle et nomade) voire d'achat (consommation à domicile mais aussi en CHR) sans s'appuyer sur un nouveau mode de production. La boisson peut aussi être consommée dans d'autres circonstances que la consommation habituelle de cidre (e.g. en dehors des repas) et par une cible plus large.
} 
Huit emballages de cidre ont ainsi été élaborés avec pour but de parvenir à un packaging crédible et réaliste et l'un d'entre eux a donc été présenté à chaque répondant afin de susciter et de mesurer ses réactions en termes d'évaluation sensorielle et d'acceptabilité.

\section{Mode d'administration, échantillon final et variables dépendantes}

Le questionnaire a été auto-administré auprès d'étudiants et de jeunes de moins de 35 ans qui représentent la principale population visée par ce type de boisson aromatisée. En réalité, huit questionnaires, correspondant aux différents traitements, ont été élaborés où seul le visuel proposé variait. Après avoir pris connaissance du visuel présentant la nouvelle boisson aromatisée, les répondants devaient répondre à des questions portant sur leur évaluation sensorielle (goût, agrément, intention de goûter, etc.), avant de finir par des questions d'ordre sociodémographiques ou portant sur leur consommation habituelle de cidre.

$\mathrm{Au}$ final, 383 individus ont répondu aux questionnaires (entre 44 et 50 individus par traitement), chiffre qui permet de garantir la normalité des distributions correspondant aux variables testées. Si l'échantillon correspond très majoritairement à une population jeune (92,4\% de moins de 24 ans et 6,3\% de 25-34 ans) et scolarisée (98,4\% d'étudiants/lycéens), il est revanche diversifié en ce qui concerne la répartition homme/femme (55,5\% de femmes).

L'ensemble des variables (Annexe 4) a été mesuré à l'aide d'items trouvés dans la littérature ou générés à la suite d'entretiens non directifs auprès d'étudiants. Les deux variables d'évaluation sensorielle ont permis chacune de mesurer par un item unique les perceptions des caractéristiques gustatives suivantes : doux et sucré. Les autres variables dépendantes ont fait l'objet d'une mesure en plusieurs items. L'agrément et la nouveauté perçue ont été mesurés à l'aide de 4 items, tandis que la pertinence perçue, l'intensité de l'ingrédient et l'intention de goûter ont fait l'objet d'une mesure en 3 items chacun. La mesure de l'implication reprend les six items proposés par Strazierri (1994). Les échelles multi-items ont fait l'objet d'une procédure de validation quantitative sur la base d'une analyse exploratoire. Les analyses factorielles ont d'abord été réalisées concept par concept, pour vérifier l'unidimensionnalité de chaque échelle. Une autre analyse factorielle (Annexe 3) incluant cette fois-ci conjointement les construits principaux ainsi que l'ensemble des variables de contrôle mesurées en plusieurs items conduit à démontrer le caractère discriminant des échelles puisque six facteurs distincts sont obtenus. Les résultats conduisent donc à accepter l'unidimensionnalité, le caractère discriminant et la fiabilité de chaque échelle avec des alphas supérieurs au taux de 0,7 hormis pour la mesure de l'intensité avec un alpha de 0,62 considéré 
comme faible mais acceptable pour une échelle en construction ${ }^{5}$. Des analyses confirmatoires ont ensuite été réalisées sous AMOS 17.0 (Annexe 5). Cette analyse permet ainsi d'établir les qualités psychométriques des échelles proposées.

\section{RESULTATS}

Effet des facteurs manipulés sur l'évaluation perceptuelle et sensorielle associée au produit Pour tester l'effet de nos facteurs manipulés sur les perceptions de pertinence, de nouveauté, d'intensité et sur les évaluations sensorielles du produit (caractère doux et sucré), nous menons une analyse de covariance multivariée (MANCOVA) où le genre, la région d'origine et l'implication sont considérées comme des variables de contrôle (co-variables). Les résultats de ce test sont présentés dans le tableau 1 et les annexes 6,7 et 8 et sont commentés ci-après. [Insérer Tableau 1 ici]

\section{L'influence de la complémentarité sur les perceptions du produit (H1)}

Les résultats confirment que la boisson associant l'arôme jugé le plus complémentaire (cassis) est considérée comme la plus pertinente ( 3.37 vs. 2.92 pour le caramel, $p=0.000$ ), validant H1a. Par ailleurs, la boisson au cassis (complémentarité élevée) est considérée comme moins nouvelle que celle au caramel (4.02 vs. 4.19, $\mathrm{p}=0.029)$, validant H1b. Aucun effet de la complémentarité de l'arôme sur les autres variables du modèle n'est décelé, amenant à rejeter les hypothèses H1c, H1d et H1e.

\section{L'influence de la prototypicalité du packaging sur l'évaluation du produit (H2)}

La prototypicalité du contenant choisi pour la boisson (bouteille ou canette) influence, au taux de $10 \%$ seulement, la douceur inférée du mélange. La boisson contenue dans une bouteille est jugée plus douce que celle présentée dans une canette (3.03 vs. 3.24, p =0.024), validant l'hypothèse H2c au seuil de $\mathbf{1 0 \%}$. Par contre, contrairement à ce qui était supposé, la prototypicalité du packaging n'influence directement ni les perceptions de pertinence, de nouveauté et d'intensité du goût ni le caractère sucré inféré amenant à rejeter les hypothèses H2a, b, d et e., Certains de ces effets sont toutefois vérifiés quand la prototypicalité du packaging interagit soit avec la complémentarité de l'arôme (effet sur l'intensité perçue, p = 0.065) soit avec la présence (ou non) de l'image de l'arôme sur le contenant (effet sur la pertinence, $\mathrm{p}=0.044$; la nouveauté, $\mathrm{p}=0.003$ et l'intensité perçue, $\mathrm{p}=0.067$ ) (Annexe 7).

\footnotetext{
${ }^{5}$ Malgré ce coefficient inférieur au seuil communément accepté, nous conservons les trois items pour couvrir entièrement le domaine du construit (validité de contenu).
} 
Ainsi, l'intensité du goût est perçue la plus faible quand le cidre est aromatisé au cassis et contenu dans une bouteille (3.18) et est la plus forte quand il est aromatisé au cassis mais contenu dans un contenant atypique, la canette (3.44). L'effet du pack est en revanche très faible quand la boisson est aromatisée au caramel (arôme peu complémentaire) (3.41 pour la canette vs. 3.46 pour la bouteille). L'effet du pack s'avère au contraire très marqué en l'absence de l'image de l'arôme sur l'étiquette (3.48 pour la canette vs. 3.22 pour la bouteille) mais peu marqué en sa présence ( 3.37 pour la canette vs. 3.42 pour la bouteille).

Ces résultats indiquent que le packaging joue un rôle majeur en ce qui concerne l'intensité perçue dans deux cas particuliers. Tout d'abord, il semble pertinent à manipuler lorsque l'arôme utilisé est complémentaire, ce qui laisse supposer que le consommateur, peu surpris par la proposition aromatique, est plus enclin à effectuer des inférences fondées sur le packaging du produit. Ensuite, le packaging devient discriminant pour l'évaluation de l'intensité quand l'image de l'arôme est absente; de nouveau nous suggérons que le consommateur choisit de focaliser son attention sur un autre attribut visuel lorsque l'information visuelle concernant l'arôme est absente.

\section{L'influence de la présence ou non de l'arôme sur les évaluations du produit (H3)}

Nous avions fait l'hypothèse que l'évaluation perceptuelle et sensorielle du produit serait différente en cas d'ajout -ou non- d'une image (i.e. présence vs. absence visuelle de l'arôme associé au cidre sur le contenant). Aucun de ces effets directs n'est vérifié, invitant à rejeter les hypothèses H3a à H3e. Globalement, en cas d'ajout de l'image de l'arôme sur l'étiquette, le mélange est jugé moins pertinent, moins nouveau, moins sucré, moins doux et le goût est perçu comme plus intense, mais aucun de ces effets n'est statistiquement significatif. Comme nous l'avons déjà souligné, la présence (vs. absence) influence cependant de manière significative la pertinence, la nouveauté et l'intensité perçues du mélange en interaction avec la prototypicalité du contenant/packaging dans lequel celui-ci est présenté.

En absence de l'image, le conditionnement le plus prototypique conduit à juger le mélange plus pertinent par opposition au conditionnement le moins prototypique. A l'inverse, lorsque l'image de l'arôme figure sur le conditionnement, ce dernier joue un rôle mineur sur l'évaluation de la pertinence. Les consommateurs semblent ainsi choisir un critère d'évaluation qui leur semble pertinent dans chacun des cas ; le packaging n'est pas utile pour évaluer la pertinence du mélange en présence de l'image de l'arôme, celle-ci fournissant suffisamment d'informations pour évaluer ce critère. En revanche, la présence de l'image et le 
conditionnement sont nécessaires à l'évaluation de la nouveauté. Lorsque l'image est présente sur le packaging, c'est le conditionnement le moins prototypique qui génère une perception de forte nouveauté. En revanche, en cas d'absence d'image, les deux conditionnements conduisent à des perceptions de nouveauté relativement proches. Ce résultat démontre l'intérêt de manipuler simultanément les variables afin de produire l'effet choisi, ici communiquer au mieux le caractère novateur du produit proposé.

\section{Un triple effet d'interaction}

L'analyse de variance décèle un effet d'interaction significatif entre les trois facteurs manipulés (cf. Annexe 8), à la fois sur la pertinence $(\mathrm{p}=0.001)$, la nouveauté $(\mathrm{p}=0.003)$ et l'intensité perçue du mélange ( $\mathrm{p}=0.005)$. Ainsi, le mélange contenant l'arôme le plus complémentaire (cassis) est jugé plus pertinent et plus nouveau présenté en bouteille sans image de l'arôme sur le pack. S'agissant du mélange associant l'arôme peu complémentaire (caramel), celui-ci est jugé plus pertinent présenté en bouteille avec présence de l'arôme sur l'étiquette et est considéré comme plus nouveau en canette que l'image de l'arôme soit présente ou non sur l'étiquette. L'information à fournir au consommateur dépend donc à la fois de l'arôme associé au produit et du contenant choisi.

Ces résultats nous permettent de proposer et d'identifier les types d'incongruence «idéale ». Les auteurs ayant travaillé sur les types d'incongruence considèrent que pour être plus efficace que la congruence en termes de mémorisation ou d'évaluation du produit ou du message , l'incongruence doit être «pertinente» mais «non attendue » (Heckler et Childers, 1992 ; Lee et Mason, 1999 ; Masterson, 2005 ; Smith, Chen et Yang, 2008). Ainsi, le mélange contenant l'arôme le plus complémentaire (cassis), jugé plus pertinent et plus nouveau lorsqu'il est présenté en bouteille sans image de l'arôme sur le pack devrait aussi susciter le plus haut niveau agrément, ce qui est confirmé par nos résultats. Pour l'arôme le moins complémentaire, l'identification du type d'incongruence «idéale » est plus complexe. Nous devons d'abord sélectionner un conditionnement jugé suffisamment nouveau par les répondants ; ici, le conditionnement le moins prototypique joue ce rôle. Ensuite, le mélange conditionné doit aussi induire un niveau de pertinence suffisamment élevé pour ne pas rendre l'incongruence trop forte, et par conséquent moins efficace (Fleck et Maille, 2010). En nous référant aux effets d'interaction (présentés en annexe 8), nous pouvons constater que lorsque l'arôme n'est pas complémentaire et que le conditionnement n'est pas prototypique, il est préférable de ne pas inclure d'image sur le packaging pour susciter une pertinence plus élevée 
et le plus fort score en termes d'agrément. Nous constatons que les combinaisons préférées correspondent dans le premier cas à un niveau d'incongruence modérée de type non attendue/pertinente et dans le second cas à un niveau d'incongruence relativement forte (peu pertinente/non attendue) posant question sur la validité de la préférence pour l'incongruence modérée (Mandler, 1982) et la pondération des deux variables dans les préférences des consommateurs.

\section{L'influence des variables de contrôle}

Ni le genre ni l'implication envers le produit (le cidre) ne s'avèrent influencer les évaluations de la boisson proposée ${ }^{6}$. En revanche, la région d'origine influence notablement la pertinence perçue du mélange $(\mathrm{p}=0.008)$, l'intensité perçue du goût $(\mathrm{p}=0.006)$ et le caractère sucré du mélange $(\mathrm{p}=0.003)$. Par ailleurs, cette variable exerce aussi un effet modérateur significatif i) sur la pertinence et l'intensité perçues du mélange en interaction avec la complémentarité de l'arôme et ii) sur le caractère sucré en interaction avec la présence/absence.

\section{Effet des évaluations perceptuelles sur l'agrément}

Nous testons ici l'effet de la pertinence perçue (H4), de la nouveauté perçue du mélange (H5) et des évaluations gustatives (sucré, doux et intensité de l'arôme, H6) sur l'agrément.

Nous validons l'existence d'une relation linéaire et positive entre la pertinence perçue du mélange et l'agrément envers le produit $(\mathbf{H 4})$, telle que visualisée sur la figure 2. Plus le mélange est jugé pertinent par les répondants, plus l'agrément envers le produit est important. [Insérer Figure 2]

Afin de montrer l'existence d'une relation non-linéaire entre la nouveauté perçue et l'agrément, il convient d'utiliser l'approche proposée par Schoormans et Robben (1997) visant à comparer les modèles de régression linéaire et quadratique pour vérifier lequel ajuste le mieux le nuage de points formé par les valeurs des variables explicatives et à expliquer. Le modèle de régression quadratique produira, en fonction de la nature du nuage de points, une courbe qui se rapprochera de la droite proposée par le modèle linéaire ou au contraire sera très marquée et dessinera une hyperbole (en forme de $\mathrm{U}$ ou de $\mathrm{U}$ inversé). La première étape pour vérifier l'existence d'une relation curvilinéaire, consiste à observer le nuage de points (ou diagramme de dispersion) ainsi que les droites et courbe d'ajustement proposés par les modèles linéaires et quadratique. Une fonction de SPSS intitulée «ajustement des fonctions » permet de comparer simultanément les ajustements proposés par les deux modèles. 
Si le modèle de régression quadratique produit une courbe, que les coefficients de premier et de second degré de l'équation sont significatifs et que la qualité de l'ajustement $\left(\mathrm{R}^{2}\right)$ est satisfaisante, alors la relation est curvilinéaire. En outre, si le coefficient de premier degré est négatif et que le coefficient de second degré est positif, la relation suit une courbe en U.

Les résultats confirment nos intuitions, le modèle de régression linéaire permettant d'obtenir un $\mathrm{R}^{2}$ de 0,02 contre 0,14 pour le modèle quadratique. Ce $\mathrm{R}^{2}$ de 0.14 est peu élevé mais cela est -au moins en partie- dû à l'étude qui est limitée à 8 produits. De plus, le coefficient de premier degré est négatif $(-0,644)$ tandis que celui de second degré positif $(0,792)$ signifiant que la relation suit une courbe en $U$, ce qui est confirmé par la courbe qui montre clairement une tendance quadratique, amenant à valider l'hypothèse H5 (Figure 3).

[Insérer figure 3]

Enfin, nous trouvons que les inférences concernant le caractère doux et l'intensité de l'arôme ont un effet direct sur l'agrément $(\mathrm{p}=0,001$ et $\mathrm{p}=0,002$ respectivement) validant partiellement H6, l'effet du caractère sucré étant quant à lui non significatif ( $\mathrm{p}=0.097)$. Cet effet est positif pour le caractère doux $(B=0.088)$ et négatif $(B=-0.117)$ pour l'intensité signifiant que plus un produit semble doux sur le plan gustatif, plus il est préféré. A l'inverse, plus l'arôme semble intense, moins les consommateurs ont tendance à apprécier le produit.

Test du rôle médiateur de la pertinence, de la nouveauté et des évaluations gustatives

Pour tester ces effets, nous suivons la démarche préconisée par Chumpitaz et Caceres (2003) ${ }^{7}$. Suite aux analyses ${ }^{8}$, la pertinence apparaît comme un médiateur complet ${ }^{9}$ de l'effet simple de la complémentarité de l'ingrédient (COMP) et de l'effet d'interaction présence/absence de l'arôme (PRES) x prototypicalité du contenant (PROT) sur l'agrément face à la boisson.

La nouveauté est aussi un médiateur complet de l'effet d'interaction entre complémentarité de l'arôme (COMP) et prototypicalité du contenant (PROT) sur l'agrément face à la boisson. La nouveauté est également un médiateur partiel $^{\mathbf{1 0}}$ de l'effet de la complémentarité de l'arôme (COMP) et de l'effet d'interaction entre présence/absence de l'arôme (PRES) et prototypicalité du contenant (PROT) sur l'agrément face à la boisson aromatisée.

\footnotetext{
${ }^{6}$ Notons qu'il n'existe également aucune différence selon que le répondant soit consommateur de cidre ou non.

${ }^{7}$ La démarche de Chumpitaz et Caceres (2003) repose sur la procédure proposée par Baron et Kenny (1986) pour analyser les processus de médiation. Notons que nous prenons également en considération les préconisations récentes de Zhao, Lynch et Chen (2011) pour examiner la médiation.

${ }^{8}$ L'ensemble des résultats des analyses des effets médiateurs sont disponibles auprès des auteurs sur demande.

${ }^{9}$ Médiation seulement indirecte selon Zhao, Lynch et Chen (2011)

${ }^{10}$ Médiation complémentaire selon Zhao, Lynch et Chen (2011)
} 
Le caractère 'sucré' n'exerce aucun effet médiateur et le caractère 'doux' est un médiateur complet de l'effet de la prototypicalité (PROT) sur l'agrément. Enfin, l'intensité de l'arôme serait un médiateur complet de l'effet d'interaction entre complémentarité de l'arôme (COMP) et prototypicalité du contenant (PROT) sur l'agrément face à la boisson aromatisée.

Effet et rôle médiateur de l'agrément sur l'intention de goûter (H7)

Nous pouvons tester simultanément l'effet simple de l'agrément sur l'intention de goûter et son effet médiateur en suivant la même procédure (Chumpitaz et Caceres 2003).

D'après les résultats obtenus, l'agrément influence l'intention de goûter $(\mathrm{p}=0.000)$ validant H7. L'agrément est aussi un médiateur complet de l'effet de la pertinence perçue, de la douceur inférée du mélange et de l'intensité perçue de l'arôme sur l'intention de goûter. L'agrément est un médiateur partiel de l'effet de la nouveauté perçue sur l'intention de goûter. Enfin, l'agrément n'exerce aucun effet médiateur en relation avec le caractère sucré.

\section{APPORTS, LIMITES ET PERSPECTIVES}

Les apports de la recherche

\section{Les apports théoriques}

D'un point de vue théorique, cette recherche se centre sur les réactions affectives et cognitives suscitées par les stimuli informationnels dans le cas d'un nouveau produit alimentaire. Elle enrichit le corpus théorique dédié aux recherches sur l'acceptabilité alimentaire en isolant deux catégories de variables susceptibles de l'améliorer. Les stimuli informationnels étudiés concernent à la fois l'aspect visuel du produit (son packaging) et l'innovation gustative. Cette recherche montre en effet que les individus infèrent des croyances en termes de pertinence, de nouveauté, de goût et d'intensité de l'arôme en fonction des caractéristiques visuelles du produit et de la complémentarité de l'arôme proposé. Le choix de l'arôme associé à la nouvelle boisson (cidre aromatisé) a un impact sur la pertinence et la nouveauté perçue du mélange. Ainsi, le cidre aromatisé au cassis (arôme le plus complémentaire) est plus pertinent et moins nouveau que le cidre aromatisé au caramel (arôme le moins complémentaire).

Cette recherche se distingue aussi des travaux antérieurs car elle introduit les conditions d'acceptation d'un packaging sortant des codes visuels de sa catégorie de produit et montre son impact sur des perceptions sensorielles majeures. La prototypicalité du packaging est en mesure de modifier les inférences gustatives, la boisson la plus prototypique conduisant les répondants à inférer un mélange plus doux. Les résultats permettent de mettre en exergue le 
rôle prépondérant du packaging en interaction avec les autres sources d'informations visuelles fournies au consommateur. Le packaging est en mesure de renforcer l'intensité de l'arôme lorsque l'image de l'arôme est absente sur le conditionnement du produit. Ces effets d'interaction illustrent la nécessité d'enrichir les travaux portant sur l'impact des variables du packaging sur les réactions du consommateur. Les travaux antérieurs portaient principalement sur la forme ou la couleur du produit (Damak, 1996 ; Lichtlé, 2002) alors que les recherches en marketing sensoriel nécessitent des approches plus complexes intégrant un nombre de variables étudiées plus important dans les protocoles expérimentaux. Multiplier les tests et les combinaisons visuelles permet d'affiner la sélection des stimuli visuels et conduit à mieux comprendre les interactions sensorielles créées par les variables manipulées.

Les résultats de cette recherche complètent les travaux de Rettie et Brewer (2000) sur le rôle de la quantité et de la place des informations sur le packaging. Les informations visuelles présentes sur le packaging peuvent jouer le rôle d'indicateurs majeurs concernant la pertinence et la nouveauté perçues de la boisson. Lorsque le packaging est prototypique, l'absence d'image permet de renforcer ou de minimiser le caractère pertinent du mélange ; à l'inverse, la présence de l'arôme est en mesure de renforcer le caractère novateur du produit lorsque le packaging est moins prototypique.

Ce travail permet enfin de mieux comprendre l'influence distincte de la pertinence et de la nouveauté sur l'agrément envers un nouveau produit alimentaire. Nous avons réussi à montrer la nature linéaire, de la relation, l'agrément augmentant avec la pertinence. En l'absence de sens entre les stimuli et le produit d'origine, l'incongruence demeure incompréhensible et détourne l'attention du produit (Edell et Staelin, 1983). La nouveauté, quant à elle, est liée à l'agrément selon une courbe en $\mathrm{U}$, les produits considérés comme peu nouveaux et très nouveaux étant préférés aux produits modérément nouveaux. L'étude de ces deux variables (pertinence et nouveauté) nous permet de proposer la combinaison d'incongruence «idéale » (à savoir très pertinente et très novatrice), celle qui génère un agrément très favorable et dès lors une intention de goûter relativement élevée. Cependant, ces résultats soulèvent également deux écueils majeurs rencontrés dans les recherches exploitant la nature bi-dimensionnelle de la congruence. Comme nous pouvons le constater, les relations entre d'une part la pertinence et l'agrément et d'autre part la nouveauté et l'agrément ne sont pas de même nature. Alors que la première est linéaire, la seconde est quadratique, il semble alors délicat de pouvoir 
classifier l'incongruence selon ces deux variables ce qui pose selon Fleck et Maille (2010) un double problème de validité interne et d'homogénéité conceptuelle.

\section{Les apports méthodologiques}

D’un point de vue méthodologique, cette recherche enrichit les études portant sur le design du packaging des produits en analysant simultanément plusieurs variables d'un prototype réaliste. Les études antérieures optaient régulièrement pour des dessins d'objets en noir et blanc (Veryzer et Hutchinson, 1998 ; Damak, 1998) ou des photographies de produits non retouchées (Hekkert et al., 2003). Ces expérimentations pouvaient nuire aux résultats en limitant d'une part la crédibilité du stimulus utilisé et d'autre part le contrôle du chercheur.

Nos prototypes ont été conçus en collaboration avec des étudiants en master innovation agroalimentaire. Le recours à des visuels réalistes et de qualité (dont la réalisation a été confiée à un infographiste) a permis aux répondants de mieux appréhender le produit et de l'évaluer comme s'il s'agissait d'un produit réellement sur le marché. L'élaboration de notre matériel expérimental s'apparente donc au processus classique de développement d'un nouveau produit (Campbell et Keller 2003) et garantit la portée opérationnelle de la recherche.

Parallèlement, l'étude simultanée de plusieurs variables permet de poursuivre les travaux entrepris en marketing sensoriel. L'évaluation du rôle des stimuli informationnels visuels (contenant et image de l'arôme) fournit une première validation empirique de l'impact des stimuli esthétiques du packaging regroupés par Magne (1999) sous la notion de 'forme design'. Les résultats de cette recherche permettent d'établir un lien entre la composante morphologique, la composante iconique et l'interaction entre ces deux composantes sur les réactions affectives et cognitives du consommateur.

\section{Les contributions managériales}

Sur le plan managérial, cette recherche permet d'apporter des indications pratiques aux gestionnaires de marque désireux de lancer de nouveaux aliments aromatisés (boisson, yaourt, eau) et fournit une démarche leur permettant de sélectionner le packaging le plus adéquat pour leurs produits. Les résultats suggèrent de considérer la complémentarité de l'arôme, la quantité d'informations visuelles et le packaging (au sens de contenant) pour générer à la fois pertinence, nouveauté et des perceptions gustatives spécifiques. Ils montrent également comment ces trois variables peuvent modifier l'intensité perçue de l'arôme proposé. Cette recherche identifie de quelle manière les composantes sensorielles visuelles jouent un rôle particulier dans l'évaluation des produits et leur acceptabilité. Elle ouvre de nouvelles pistes 
de positionnement sur des marchés alimentaires où l'offre est encombrée et montre l'impact des composantes visuelles sur la transmission de messages spécifiques.

Enfin, en testant l'impact de la pertinence et de la nouveauté sur l'agrément envers le produit et l'intention de le goûter, il est possible d'évaluer l'impact distinctif de ces deux variables dans le processus de choix des consommateurs et leurs pondérations respectives. Nous sommes également en mesure d'indiquer les stimuli visuels les plus promptes à véhiculer ces deux perceptions (pertinence et nouveauté). L'apparence visuelle est le premier vecteur d'innovation (Radford et Bloch 2011). Si le produit est capable de transmettre ce message par les différentes variables qui composent l'apparence visuelle du produit, il sera remarqué par les consommateurs qui le distingueront alors de ses concurrents.

\section{Limites et perspectives}

Cette recherche comporte des limites qui ouvrent autant de voies de recherches. Une première limite tient au caractère particulier du produit étudié ici (cidre aromatisé) qui pose alors la question de la généralisation possible des résultats à d'autres produits issus de la même catégorie (boisson) ou d'une autre catégorie. Une première voie de recherche serait de dupliquer l'étude sur d'autres produits. Par ailleurs, de nombreuses variables susceptibles d'influencer les croyances et l'évaluation du produit par le consommateur ne sont pas prises en compte dans ce modèle, qu'il s'agisse d'éléments manipulables (ex : nom de marque, typographie) ou de variables médiatrices (ex : authenticité perçue). Concernant les éléments manipulables, la typographie nous semble particulièrement intéressante. Certaines polices seraient plus appropriées aux innovations en fonction des catégories de produits étudiées (Celhay, 2010). Dans le cas du cidre aromatisé, nous pourrions tester plusieurs typographies et tailles de police afin de connaître la combinaison qui renforcera l'acceptation de cette nouvelle boisson. Afin de compléter la présente recherche, nous envisageons aussi de modifier la couleur de la bouteille ou de la canette. Nous vérifierons si le contenant de couleur violette dans le cas du cassis est susceptible de renforcer l'arôme utilisé ou si à l'inverse, la couleur entraine un sentiment de rejet de la part des consommateurs en raison de la perception d'un goût trop prononcé. Les expérimentations futures permettront également de faire varier l'intensité de la couleur utilisée afin de mettre en avant le potentiel des deux autres dimensions de la couleur, à savoir la luminosité et la saturation. Elles pourront aussi comprendre des images différentes afin de comparer les effets de la présentation des arômes, 
par exemple des grains de cassis dispersés et non en baies ou du caramel liquide à la place des morceaux présentés ici.

Cette recherche pourrait également intégrer des variables modératrices. Lenglet (2007) met en avant l'impact modérateur des tendances exploratoires afin de comprendre le goût pour les aliments. Les préférences sensorielles dépendraient de variables personnelles telles que la recherche de variété alimentaire définie comme « le facteur de motivation qui a pour but d'apporter une variation dans la stimulation grâce à une consommation variée de produits alimentaires, indépendamment de la valeur fonctionnelle ou instrumentale des différentes alternatives »(Van Trijp et Steenkamp, 1992; p.184). Les individus ayant une forte tendance à la recherche de variété sont plus aptes à accepter les innovations. Le besoin d'être unique aurait quant à lui pour conséquence de vouloir posséder des produits innovants et surprenants et d'adopter un comportement dissemblable aux autres (Irmak, Vallen et Sen, 2010).

Par ailleurs, il serait intéressant d'identifier si les inférences identifiées sont susceptibles d'engendrer des différences comportementales en termes d'achat et de prix à payer. Jaeger et Harker (2005) montrent que les consommateurs sont prêts à payer presque deux fois plus cher un kiwi dont la chair est de couleur rose, ce prix étant deux fois et demi plus élevé lorsque l'on indique que ce kiwi n'est pas génétiquement modifié. La couleur du produit, la nouveauté perçue et les stimuli informationnels (mention OGM ou non) ont un impact sur le prix à payer encourageant les industriels à proposer des nouveautés sur le marché.

Ensuite, poursuivre l'analyse par une évaluation sensorielle permettrait de confronter l'évaluation basée sur les attributs extrinsèques du produit et l'évaluation gustative basée sur ses propriétés organoleptiques. Les résultats permettraient d'identifier les différences de perception et d'analyser les raisons d'une confirmation ou disconfirmation des attentes avec leur impact corollaire sur la satisfaction. Une voie plus prometteuse consisterait à recourir à un plan imbriqué ('nested factors') en prenant comme facteur de 'branchement' ('branching factor') soit l'arôme soit le contenant. Un tel design aurait l'avantage d'accroitre la validité de notre recherche mais est difficile à mettre en place du fait du nombre limité de recherches utilisant cette méthode. 
A1. Résumé des hypothèses et de leur validation

\begin{tabular}{|c|c|c|c|}
\hline VD & VI & Effets attendus & Résultat \\
\hline \multicolumn{4}{|l|}{ Manova } \\
\hline \multirow{7}{*}{ Pertinence } & Complémentarité (H1a) & $\operatorname{Comp}(+)>\operatorname{Comp}(-)$ & Confirmée \\
\hline & Prototypicalité (H2a) & $\operatorname{Prot}(+)>\operatorname{Prot}(-)$ & n.s. \\
\hline & Présence/Absence (H3a) & Présence > Absence & n.s. \\
\hline & Comp x Prot & Comp (+) x Prot $(+)>\operatorname{Comp}(-) \times \operatorname{Prot}(-)$ & n.s. \\
\hline & Comp x Abs/Pres & Comp (+) x Pres $>$ Comp (-) x Abs & n.s. \\
\hline & Prot x Abs/Pres & Prot $(+) \times$ Pres $>$ Prot $(-) \times$ Abs & Confirmée \\
\hline & Comp x Prot x Abs/Pres & Comp $(+) \times$ Prot $(+) \times$ Pres $>$ autres combinaisons & Confirmée \\
\hline \multirow{7}{*}{ Nouveauté } & Complémentarité (H1b) & Comp $(+)<\operatorname{Comp}(-)$ & Confirmée \\
\hline & Prototypicalité (H2b) & Prot $(+)<\operatorname{Prot}(-)$ & n.s. \\
\hline & Présence/Absence (H3b) & Presence > Absence & n.s. \\
\hline & Comp x Prot & Comp (-) x Prot (-) > autres combinaisons & Confirmée \\
\hline & Comp x Abs/Pres & Comp (-) x Présence > autres combinaisons & n.s. \\
\hline & Prot x Abs/Pres & Prot (-) x Présence > autres combinaisons & Confirmée \\
\hline & Comp x Prot x Abs/Pres & Comp (-) x Prot (-) x Pres $>$ autres combinaisons & Confirmée \\
\hline \multirow{7}{*}{ Doux } & Complémentarité (H1c) & Comp $(+)>\operatorname{Comp}(-)$ & n.s. \\
\hline & Prototypicalité (H2c) & Prot $(+)>\operatorname{Prot}(-)$ & Confirmée \\
\hline & Présence/Absence (H3c) & Presence > Absence & n.s. \\
\hline & Comp x Prot & Comp (+) x Prot $(+)>\operatorname{Comp}(-) \times \operatorname{Prot}(-)$ & n.s. \\
\hline & Comp x Abs/Pres & Comp (+) x Pres > Comp (-) x Abs & n.s. \\
\hline & Prot x Abs/Pres & Prot $(+)$ x Pres $>$ Prot $(-)$ x Abs & n.s. \\
\hline & Comp x Prot x Abs/Pres & Comp $(+) \times$ Prot $(+) \times$ Pres $>$ autres combinaisons & n.s. \\
\hline \multirow{7}{*}{ Sucré } & Complémentarité (H1d) & Comp $(+)>\operatorname{Comp}(-)$ & n.s. \\
\hline & Prototypicalité (H2d) & $\operatorname{Prot}(+)>\operatorname{Prot}(-)$ & n.s. \\
\hline & Présence/Absence (H3d) & Presence > Absence & n.s. \\
\hline & Comp x Prot & Comp (+) x Prot $(+)>\operatorname{Comp~(-)~x~Prot~(-)~}$ & n.s. \\
\hline & Comp x Abs/Pres & Comp (+) x Pres > Comp (-) x Abs & n.s. \\
\hline & Prot x Abs/Pres & Prot $(+)$ x Pres $>$ Prot $(-)$ x Abs & n.s. \\
\hline & Comp x Prot x Abs/Pres & Comp $(+) \times$ Prot $(+) \times$ Pres $>$ autres combinaisons & n.s. \\
\hline \multirow[t]{5}{*}{ Intensité } & Complémentarité (H1e) & Comp $(+)>\operatorname{Comp}(-)$ & n.s. \\
\hline & Prototypicalité (H2e) & Prot $(+)>\operatorname{Prot}(-)$ & n.s. \\
\hline & Présence/Absence (H3e) & Presence > Absence & n.s. \\
\hline & Comp x Prot & $\operatorname{Comp}(+) \times \operatorname{Prot}(+)>\operatorname{Comp}(-) \times \operatorname{Prot}(-)$ & Confirmée \\
\hline & Comp x Abs/Pres & Comp (+) x Pres $>$ Comp (-) x Abs & n.s. \\
\hline
\end{tabular}




\begin{tabular}{|l|l|l|l|}
\hline & Prot x Abs/Pres & Prot (+) x Pres > Prot (-) x Abs & Confirmée \\
\cline { 2 - 4 } & Comp x Prot x Abs/Pres & Comp (+) x Prot (+) x Pres > autres combinaisons & Confirmée \\
\hline \multicolumn{2}{|l|}{ Régressions } & \multicolumn{2}{|l|}{} \\
\hline Pertinence & Agrément (H4) & Pert (+) > Pert (-) (relation linéaire) & Confirmée \\
\hline Nouveauté & Agrément (H5) & $\begin{array}{l}\text { Nouv (+) et Nouv (-) > Nouveauté modérée } \\
\text { courbe en U) }\end{array}$ & Confirmée \\
\hline Doux & Agrément (H6) & Doux (+) ? Doux (-) & Doux (+) > Doux (-) \\
\hline Sucré & Agrément (H6) & Sucré (+) ? Sucré (-) & n.s. \\
\hline Intensité & Agrément (H6) & Intensité (+) ? Intensité (-) & $\begin{array}{l}\text { Intensité (+) }< \\
\text { Intensité (-) }\end{array}$ \\
\hline Agrément & Intention de goûter (H7) & Agrément (+) > Agrément (-) & Confirmée \\
\hline
\end{tabular}


A2.Quatre versions testées de cidre aromatisé parmi les huit possibles

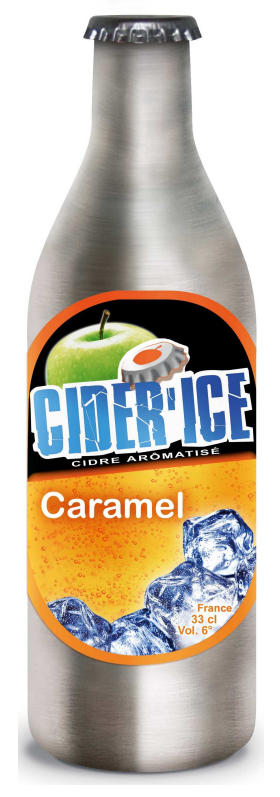

Version Bouteille / Caramel Absence ingrédient

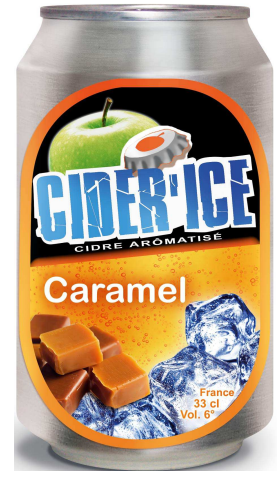

Version Canette caramel Présence ingrédient

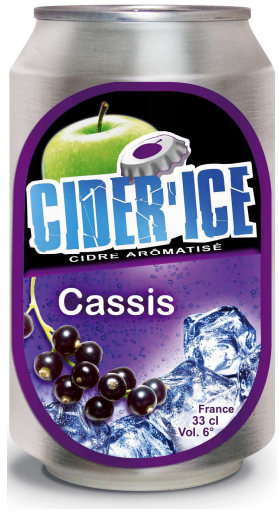

Version Canette / Cassis

Présence ingrédient

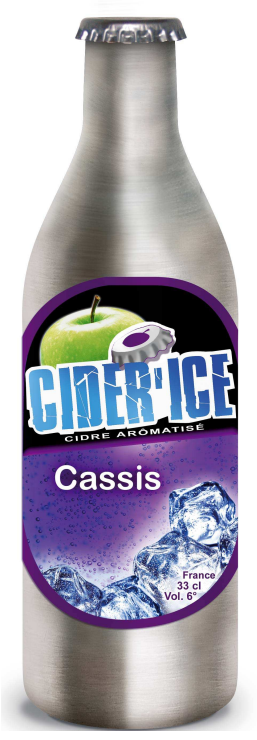

Version Bouteille / Cassis Absence ingrédient 
A3. Résultats analyse factorielle exploratoire

\begin{tabular}{|c|c|c|c|c|c|c|c|c|c|}
\hline \multirow[b]{2}{*}{ Construits } & \multirow[b]{2}{*}{ Items } & \multicolumn{6}{|c|}{ Facteurs } & \multicolumn{2}{|c|}{ Valeurs propres } \\
\hline & & Pert. & Agrém. & Impl. & Nouv. & IG & Intensité & Total & $\begin{array}{c}\% \text { de } \\
\text { Variance }\end{array}$ \\
\hline $\begin{array}{c}\text { Pertinence } \\
(\text { alpha }=0,84)\end{array}$ & $\begin{array}{l}\text { PERT1 } \\
\text { PERT2 } \\
\text { PERT3 } \\
\end{array}$ & $\begin{array}{l}0,827 \\
0,805 \\
0,785 \\
\end{array}$ & & & & & & 2,289 & 10,899 \\
\hline $\begin{array}{c}\text { Agrément } \\
(\text { alpha }=0,79)\end{array}$ & $\begin{array}{l}\text { AGR2 } \\
\text { AGR3 } \\
\text { AGR4 } \\
\text { AGR1 }\end{array}$ & & $\begin{array}{l}, 763 \\
, 706 \\
, 679 \\
, 670 \\
\end{array}$ & & & 0,300 & & 3,351 & 15,960 \\
\hline $\begin{array}{l}\text { Implication } \\
\text { (alpha }=0,91)\end{array}$ & $\begin{array}{l}\text { IMP5 } \\
\text { IMP2 } \\
\text { IMP4 } \\
\text { IMP1 } \\
\text { IMP3 } \\
\text { IMP6 }\end{array}$ & & & $\begin{array}{l}, 884 \\
, 876 \\
, 855 \\
, 849 \\
, 810 \\
, 737\end{array}$ & & & & 4,322 & 20,582 \\
\hline $\begin{array}{c}\text { Nouveauté } \\
\text { (alpha =0,76) }\end{array}$ & $\begin{array}{c}\text { NOUV1 } \\
\text { NOUV2 } \\
\text { (inv.) } \\
\text { NOUV3 } \\
\text { NOUV4 }\end{array}$ & & & & $\begin{array}{l}0,786 \\
0,773 \\
0,731 \\
0,704 \\
\end{array}$ & & & 2,289 & 10,899 \\
\hline $\begin{array}{c}\text { Intention de } \\
\text { goûter } \\
(\text { alpha }=0,92)\end{array}$ & $\begin{array}{l}\text { IG1 } \\
\text { IG2 } \\
\text { IG3 }\end{array}$ & & $\begin{array}{l}0.319 \\
0.329 \\
0,320\end{array}$ & & & $\begin{array}{l}0,753 \\
0,739 \\
0,662\end{array}$ & & 2,289 & 10,899 \\
\hline $\begin{array}{c}\text { Intensité } \\
(\text { alpha }=0,62)\end{array}$ & $\begin{array}{l}\text { INT1 } \\
\text { INT2 } \\
\text { INT3 } \\
\end{array}$ & $-0,352$ & & & & & $\begin{array}{l}0,724 \\
0,673 \\
0,659 \\
\end{array}$ & 1,424 & 6,779 \\
\hline
\end{tabular}


A4. Instruments de mesure

\begin{tabular}{|c|c|c|}
\hline Variable & Affirmations & Format \\
\hline \multirow{3}{*}{$\begin{array}{l}\text { Intention de } \\
\text { Goûter }\end{array}$} & J'aimerais goûter ce cidre aromatisé & \multirow{25}{*}{ Lickert 1 à 5} \\
\hline & $\begin{array}{l}\text { Je ne serais pas inquiet(e) à l'idée de goûter ce cidre } \\
\text { aromatisé }\end{array}$ & \\
\hline & Je suis curieux à l'idée de goûter ce cidre aromatisé & \\
\hline \multirow{4}{*}{ Agrément } & Ce mélange semble agréable & \\
\hline & Ce mélange est plaisant & \\
\hline & Cette boisson semble agréable en bouche & \\
\hline & Ce cidre aromatisé me plait & \\
\hline \multirow{3}{*}{ Intensité } & Le goût du cidre semble masqué & \\
\hline & Ce n'est plus du cidre & \\
\hline & Cet arôme semble masquer le goût du cidre & \\
\hline Doux & Le goût de cette boisson semble doux & \\
\hline Sucré & Cette boisson semble sucrée & \\
\hline \multirow{6}{*}{ Implication } & Le cidre est produit qui compte beaucoup pour moi & \\
\hline & $\begin{array}{l}\text { Le cidre est un produit auquel j'accorde une } \\
\text { importance particulière }\end{array}$ & \\
\hline & J'aime particulièrement parler de cidre & \\
\hline & On peut dire que le cidre est produit qui m'intéresse & \\
\hline & Je me sens particulièrement attiré(e) par le cidre & \\
\hline & $\begin{array}{l}\text { Le seul fait de me renseigner sur le cidre, pour en } \\
\text { acheter, est un plaisir }\end{array}$ & \\
\hline \multirow{3}{*}{ Pertinence } & Le cassis (caramel) se marie bien avec le cidre & \\
\hline & Cela semble être un bon mélange & \\
\hline & Ce sont deux saveurs qui s'allient bien & \\
\hline \multirow{4}{*}{ Nouveauté } & Cette boisson semble novatrice & \\
\hline & J'ai déjà vu ce type de cidre (inv.) & \\
\hline & Ce mélange est inédit & \\
\hline & Cette boisson semble originale & \\
\hline Fréquence & A quelle fréquence consommez-vous du cidre ? & $\begin{array}{l}\text { Ordonnée de } \\
\text { tous les jours } \\
\text { (1) à jamais (5) }\end{array}$ \\
\hline Format & $\begin{array}{l}\text { En général sous quel 'format' consommez-vous le } \\
\text { cidre? }\end{array}$ & $25 / 33 / 75 \mathrm{cl}$ \\
\hline $\begin{array}{l}\text { Région } \\
\text { d'origine }\end{array}$ & Dans quelle région êtes vous né(e) ? & $\begin{array}{l}26 \text { régions } \\
+ \text { autres }\end{array}$ \\
\hline Genre & Vous êtes ... & $\mathrm{H} / \mathrm{F}$ \\
\hline
\end{tabular}


A5. Résultats des analyses confirmatoires

Les variables de pertinence (PERT), nouveauté (NOUV), intensité (INT), agrément (AGR), intention de gouter (IG) et d'implication (IMPL) ont été considérées simultanément. Ce modèle présente des indices d'ajustement satisfaisants $\left(\chi^{2} / \mathrm{dl}=1,992 ; \mathrm{GFI}=0,903\right.$; $\mathrm{AGFI}=0,875 ; \mathrm{RMSEA}=0,051)$. La fiabilité des échelles de mesure est également satisfaisante avec des coefficients de cohérence interne $(\rho)$ de : PERT $=0,859$, NOUV=0,789, $\mathrm{INT}=0,738, \mathrm{AGR}=0,811, \mathrm{IG}=0,911$ et $\mathrm{IMPL}=0,930$. Concernant la validité convergente, les coefficients $(\rho V C)$ sont acceptables pour tous ces construits : PERT $=0,670$ et NOUV $=0,509, \mathrm{INT}=0,479, \mathrm{AGR}=0,522, \mathrm{IG}=0,774$ et $\mathrm{IMPL}=0,692$. La validité discriminante est enfin établie, le coefficient de validité convergente étant supérieur au carré du coefficient de corrélation entre les construits $\left(\mathrm{r}^{2}{ }_{\mathrm{ij}}\right)$.

\begin{tabular}{|c|c|c|c|c|c|}
\hline \multirow{2}{*}{ VARIABLE } & \multirow{2}{*}{$\begin{array}{c}\mathrm{Nb} \\
\text { d'items }\end{array}$} & \multicolumn{2}{|c|}{ Fiabilité } & \multirow{2}{*}{$\begin{array}{c}\text { Validité } \\
\text { convergente } \\
(\rho v c>0,5)\end{array}$} & \multirow{2}{*}{$\begin{array}{c}\text { Validité } \\
\text { Discriminante } \\
\left(\rho v c>r^{2}{ }_{i j}\right)\end{array}$} \\
\hline & & $\alpha$ & Rhô & & \\
\hline Pertinence (PERT) & 3 & 0,84 & 0,859 & 0,670 & $\begin{array}{c}0,670> \\
{[0,001-0,401]}\end{array}$ \\
\hline $\begin{array}{l}\text { Nouveauté } \\
\text { (NOUV) }\end{array}$ & 4 & 0,76 & 0,789 & 0,509 & $\begin{array}{c}0,509> \\
{[0,001-0,056]}\end{array}$ \\
\hline Intensité (INT) & 3 & 0,62 & 0,718 & 0,479 & $\begin{array}{c}0,479> \\
{[0,002-0,399]}\end{array}$ \\
\hline Agrément (AGR) & 4 & 0,79 & 0,811 & 0,522 & $\begin{array}{c}0,522> \\
{[0,001-0,401]}\end{array}$ \\
\hline $\begin{array}{l}\text { Intention de Goûter } \\
\text { (IG) }\end{array}$ & 3 & 0,92 & 0,911 & 0,774 & $\begin{array}{c}0,774> \\
{[0,065-0,371]}\end{array}$ \\
\hline Implication (IMP) & 6 & 0,91 & 0,930 & 0,692 & $\begin{array}{c}0,692> \\
{[0,001-0,065]}\end{array}$ \\
\hline
\end{tabular}

\begin{tabular}{|c|c|c|c|c|c|}
\hline $\mathrm{r}_{\mathrm{ij}}^{2}$ & INT & IG & IMP & AGR & NOUV \\
\hline & & & & & \\
\hline IG & 0,088 & & & & \\
\hline IMP & 0,002 & 0,065 & & & \\
\hline AGR & 0,278 & 0,371 & 0,000 & & \\
\hline NOUV & 0,024 & 0,056 & 0,001 & 0,025 & \\
\hline PERT & 0,399 & 0,225 & 0,001 & 0,401 & 0,001 \\
\hline
\end{tabular}


A6. Moyennes pour les facteurs manipulés (Complémentarité de l'arôme, Présence de l'image de l'arôme, Prototypicalité du Contenant)

\begin{tabular}{|c|c|c|c|c|c|c|c|c|}
\hline \multirow{2}{*}{ Complémentarité } & \multirow{2}{*}{ Présence } & \multirow{2}{*}{ Prototypicalité } & Pertinence & Nouveauté & Sucré & Doux & Intensité & \multirow{2}{*}{$\mathrm{N}$} \\
\hline & & & Moyenne & Moyenne & Moyenne & Moyenne & Moyenne & \\
\hline \multirow[t]{9}{*}{ Forte } & Sans & Faible & 3,10 & 3,84 & 4,39 & 2,92 & 3,64 & 49 \\
\hline & & Forte & 3,83 & 4,34 & 4,41 & 3,56 & 2,99 & 48 \\
\hline & & Total & 3,47 & 4,09 & 4,39 & 3,24 & 3,32 & 97 \\
\hline & Avec & Faible & 3,41 & 4,18 & 4,26 & 3,09 & 3,24 & 43 \\
\hline & & Forte & 3,15 & 3,73 & 4,11 & 3,10 & 3,37 & 46 \\
\hline & & Total & 3,28 & 3,96 & 4,18 & 3,10 & 3,30 & 89 \\
\hline & Total & Faible & 3,28 & 4,01 & 4,32 & 3,00 & 3,44 & 92 \\
\hline & & Forte & 3,49 & 4,03 & 4,26 & 3,33 & 3,18 & 94 \\
\hline & & Total & 3,37 & 4,02 & 4,29 & 3,17 & 3,31 & 186 \\
\hline \multirow[t]{9}{*}{ Faible } & Sans & Faible & 2,95 & 4,31 & 4,34 & 3,01 & 3,32 & 47 \\
\hline & & Forte & 2,86 & 4,05 & 4,01 & 3,09 & 3,44 & 48 \\
\hline & & Total & 2,90 & 4,18 & 4,17 & 3,05 & 3,38 & 95 \\
\hline & Avec & Faible & 2,85 & 4,31 & 4,23 & 3,12 & 3,50 & 47 \\
\hline & & Forte & 3,03 & 4,09 & 4,22 & 3,23 & 3,47 & 46 \\
\hline & & Total & 2,94 & 4,20 & 4,22 & 3,17 & 3,49 & 93 \\
\hline & Total & Faible & 2,90 & 4,31 & 4,28 & 3,06 & 3,41 & 94 \\
\hline & & Forte & 2,94 & 4,07 & 4,11 & 3,16 & 3,46 & 94 \\
\hline & & Total & 2,92 & 4,19 & 4,20 & 3,11 & 3,43 & 188 \\
\hline \multirow[t]{9}{*}{ Total } & Sans & Faible & 3,02 & 4,08 & 4,36 & 2,96 & 3,48 & 96 \\
\hline & & Forte & 3,34 & 4,20 & 4,21 & 3,32 & 3,22 & 96 \\
\hline & & Total & 3,18 & 4,14 & 4,28 & 3,14 & 3,35 & 192 \\
\hline & Avec & Faible & 3,13 & 4,25 & 4,24 & 3,10 & 3,37 & 90 \\
\hline & & Forte & 3,09 & 3,91 & 4,16 & 3,16 & 3,42 & 92 \\
\hline & & Total & 3,11 & 4,08 & 4,20 & 3,13 & 3,40 & 182 \\
\hline & Total & Faible & 3,08 & 4,16 & 4,30 & 3,03 & 3,43 & 186 \\
\hline & & Forte & 3,21 & 4,05 & 4,19 & 3,24 & 3,32 & 188 \\
\hline & & Total & 3,14 & 4,10 & 4,24 & 3,14 & 3,37 & 374 \\
\hline
\end{tabular}


A7. Effets d'interaction à deux niveaux

Moyennes Intensité pour les effets Complémentarité x Prototypicalité du Pack et Prototypicalité du Pack x Présence (vs. abs.)
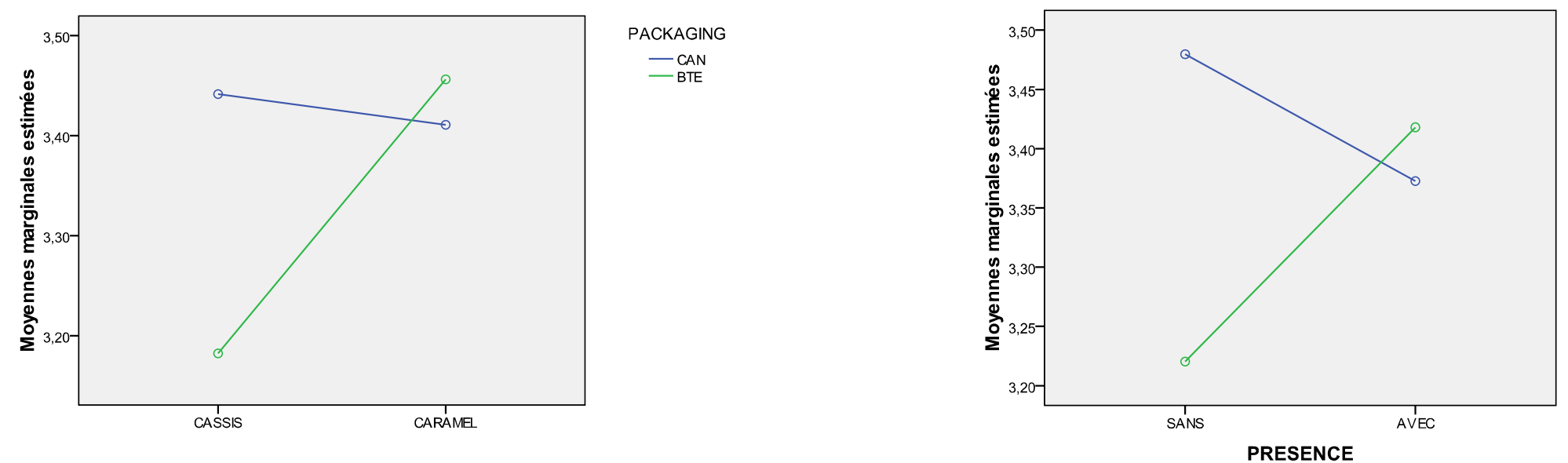

PACKAGING 二

Moyennes Pertinence et Moyennes Nouveauté pour l'effet Prototypicalité du Pack x Présence (vs. abs.)
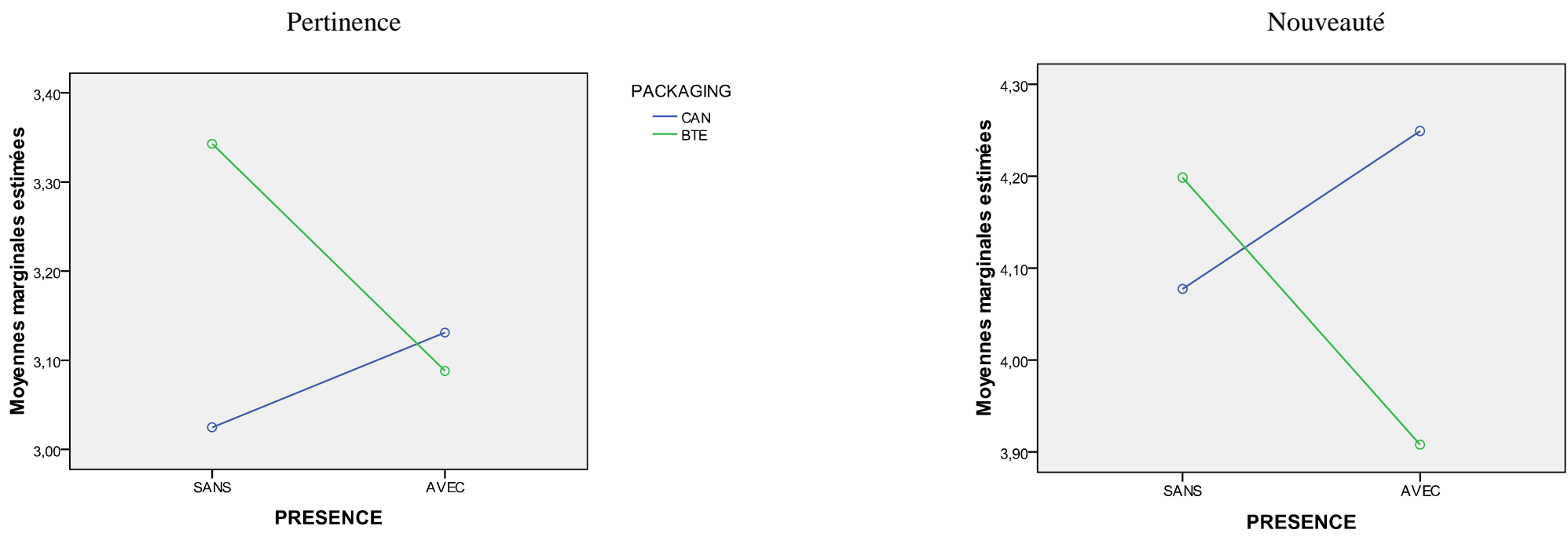

PACKAGING

PACKAGING

二 CAN

PRESENCE 
A8. Effets d'interaction à trois niveaux

Moyennes Pertinence pour l'effet Complémentarité x Prototypicalité du Pack x Présence (vs. abs.)

CASSIS

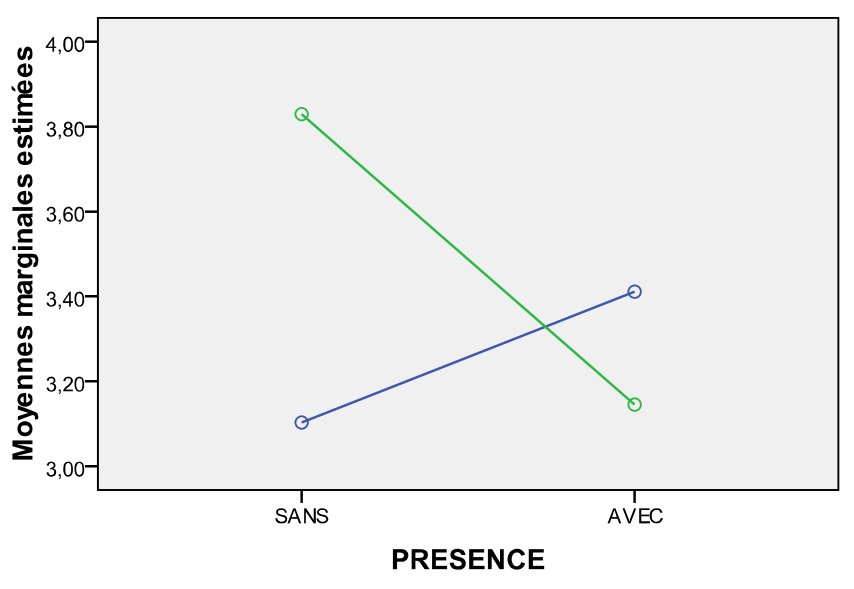

CARAMEL

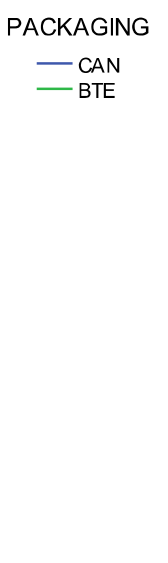

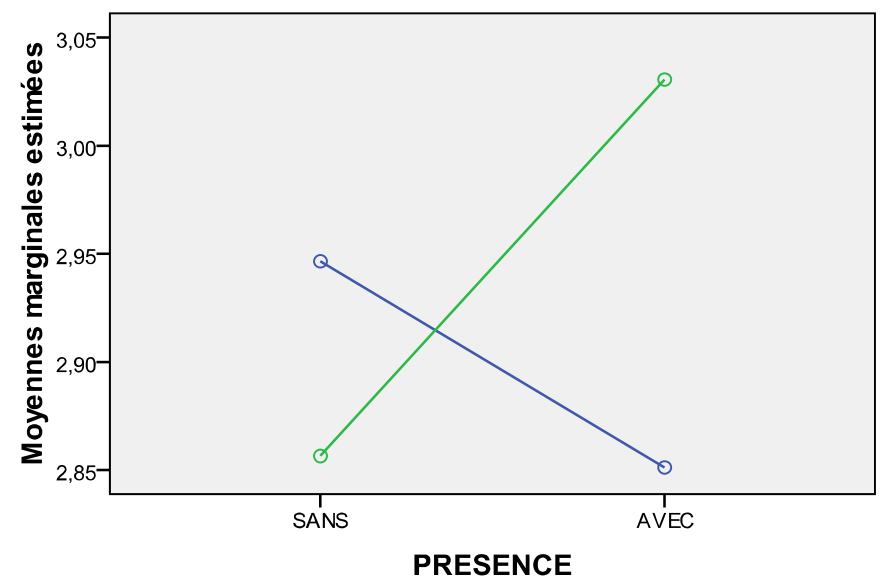

PRESENCE
PACKAGING

二 $\mathrm{CAN}$

Moyennes Nouveauté pour l'effet Complémentarité x Prototypicalité du Pack x Présence (vs. abs.)

CASSIS

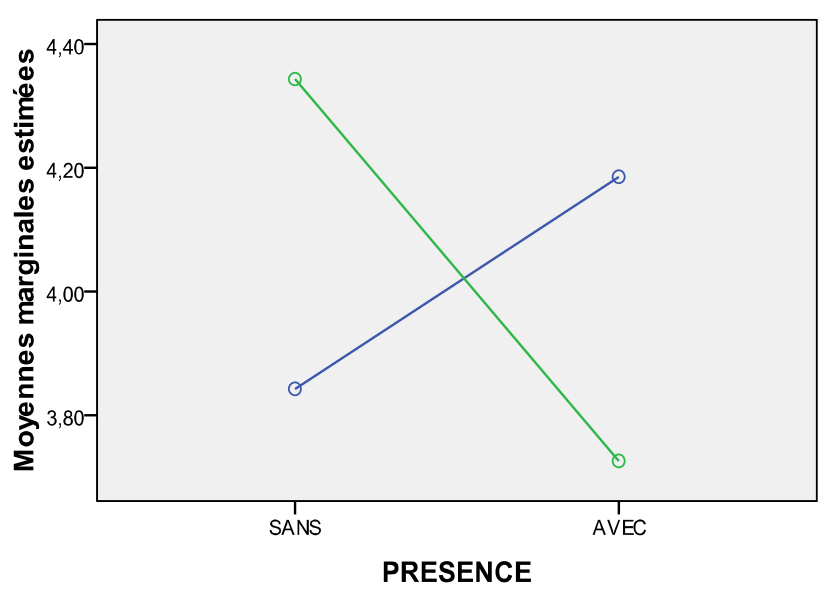

CARAMEL

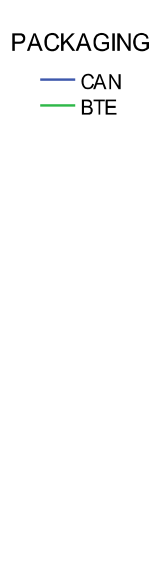

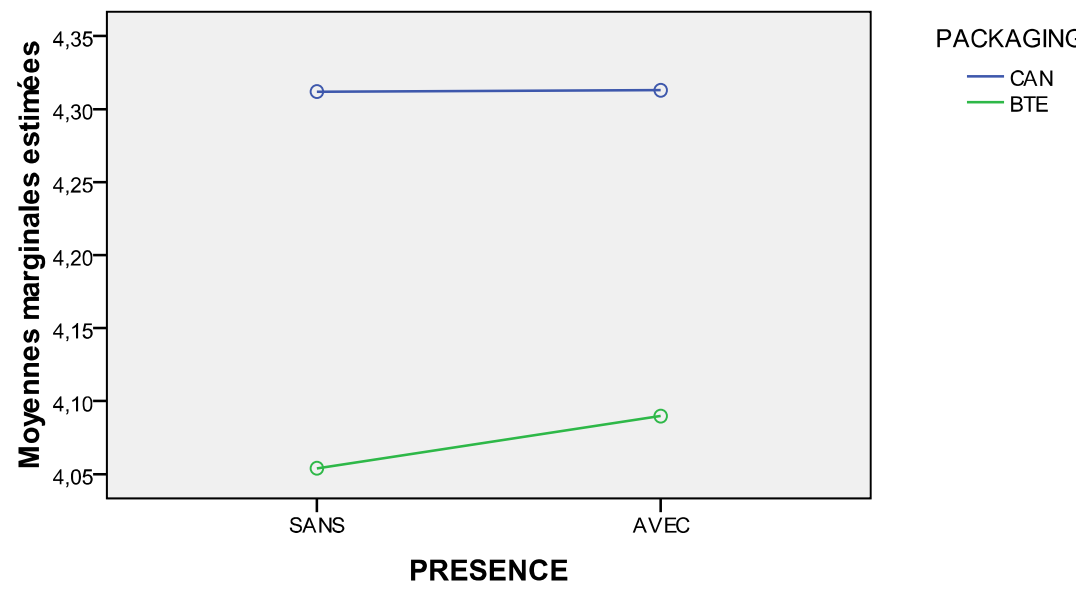


TABLEAUX

Tableau 1. Résultats de la MANCOVA (Test des Hypothèses H1 à H3)

\begin{tabular}{|c|c|c|c|c|c|c|c|}
\hline Source & $\begin{array}{l}\text { Variable } \\
\text { dépendante }\end{array}$ & $\begin{array}{c}\text { Somme des } \\
\text { carrés de } \\
\text { type III } \\
\end{array}$ & ddl & $\begin{array}{c}\text { Moyenne } \\
\text { des } \\
\text { carrés }\end{array}$ & $\mathrm{D}$ & Sig. & Conclusion \\
\hline \multirow{5}{*}{$\begin{array}{l}\text { COMPLEMENTARITE } \\
\text { DE L'AROME } \\
\text { (faible vs. forte) }\end{array}$} & PERT & 18,250 & 1 & 18,250 & 25,699 &, $000^{* * *}$ & $\begin{array}{c}\text { H1 a } \\
\text { VALIDEE }\end{array}$ \\
\hline & NOUV & 2,528 & 1 & 2,528 & 4,819 &, $029 * *$ & $\begin{array}{c}\text { H1 b } \\
\text { VALIDEE }\end{array}$ \\
\hline & Sucré & ,754 & 1 & ,754 & 1,232 & ,268 & \\
\hline & Doux & ,301 & 1 & ,301 & 259 & ,611 & \\
\hline & Intensité & 1,331 & 1 & 1,331 & 2,175 & , 141 & \\
\hline \multirow{5}{*}{$\begin{array}{l}\text { PROTOTYPICALITE } \\
\text { DU CONTENANT } \\
\text { (faible) }\end{array}$} & PERT & 1,686 & 1 & 1,686 & 2,374 & 124 & $\begin{array}{l}\text { H2 a NON } \\
\text { VALIDEE }\end{array}$ \\
\hline & NOUV & 1,081 & 1 & 1,081 & 2,061 &, 152 & $\begin{array}{l}\text { H2 b NON } \\
\text { VALIDEE }\end{array}$ \\
\hline & Sucré & 1,181 & 1 & 1,181 & 1,930 & ,166 & \multirow{3}{*}{$\begin{array}{c}H 2 c \\
\text { VALIDEE } \\
(10 \%)\end{array}$} \\
\hline & Doux & 3,989 & 1 & 3,989 & 3,433 &, $065 *$ & \\
\hline & Intensité & 1,021 & 1 & 1,021 & 1,669 & ,197 & \\
\hline \multirow{5}{*}{$\begin{array}{l}\text { PRESENCE DE } \\
\text { L'IMAGE DE } \\
\text { L'AROME } \\
\text { (vs. absence) }\end{array}$} & PERT & ,486 & 1 & ,486 & ,685 & ,408 & \multirow{5}{*}{$\begin{array}{l}\text { H3 NON } \\
\text { VALIDEE }\end{array}$} \\
\hline & NOUV &, 311 & 1 &, 311 &, 593 & ,442 & \\
\hline & Sucré & ,591 & 1 & ,591 & ,966 &, 326 & \\
\hline & Doux & ,007 & 1 & ,007 & ,006 & ,938 & \\
\hline & Intensité & , 181 & 1 &, 181 & ,296 & ,587 & \\
\hline \multirow{5}{*}{$\begin{array}{l}\text { COMPLEMENTARITE } \\
\text { * PROTOTYPICALITE }\end{array}$} & PERT & ,773 & 1 & ,773 & 1,089 & ,297 & \multirow{5}{*}{$\begin{array}{c}2 \text { Effets } \\
\text { d'interaction } \\
\text { significatifs à } \\
10 \%\end{array}$} \\
\hline & NOUV & 1,535 & 1 & 1,535 & 2,926 & $088^{*}$ & \\
\hline & Sucré & ,248 & 1 & ,248 & ,405 & ,525 & \\
\hline & Doux & 1,191 & 1 & 1,191 & 1,025 & ,312 & \\
\hline & Intensité & 2,089 & 1 & 2,089 & 3,414 &, $065^{*}$ & \\
\hline \multirow{5}{*}{$\begin{array}{l}\text { COMPLEMENTARITE } \\
\text { * PRESENCE }\end{array}$} & PERT & 1,134 & 1 & 1,134 & 1,597 &, 207 & \\
\hline & NOUV &, 532 & 1 &, 532 & 1,014 &, 315 & \\
\hline & Sucré & 1,499 & 1 & 1,499 & 2,449 & , 118 & \\
\hline & Doux & 1,496 & 1 & 1,496 & 1,288 & ,257 & \\
\hline & Intensité & ,308 & 1 & ,308 & ,504 & ,478 & \\
\hline \multirow{5}{*}{$\begin{array}{l}\text { PRESENCE } * \\
\text { PROTOTYPICALITE }\end{array}$} & PERT & 2,901 & 1 & 2,901 & 4,085 &, $044^{* *}$ & \multirow{2}{*}{$\begin{array}{c}\text { Effets } \\
\text { d'interaction } \\
\text { significatifs }\end{array}$} \\
\hline & NOUV & 4,764 & 1 & 4,764 & 9,083 &, $003^{* * *}$ & \\
\hline & Sucré &, 124 & 1 &, 124 & ,202 & ,654 & \\
\hline & Doux & 2,052 & 1 & 2,052 & 1,766 &, 185 & \\
\hline & Intensité & 2,067 & 1 & 2,067 & 3,377 &, $067^{*}$ & $\begin{array}{l}\text { Effet d'inter. } \\
\text { sig. à } 10 \%\end{array}$ \\
\hline
\end{tabular}




\begin{tabular}{|c|c|c|c|c|c|c|c|}
\hline Source (suite) & $\begin{array}{l}\text { Variable } \\
\text { dépendante }\end{array}$ & $\begin{array}{l}\text { Somme des } \\
\text { carrés de } \\
\text { type III }\end{array}$ & ddl & $\begin{array}{c}\text { Moyenne } \\
\text { des } \\
\text { carrés }\end{array}$ & $\mathrm{D}$ & Sig. & Conclusion \\
\hline \multirow{5}{*}{$\begin{array}{l}\text { COMPLEMENTARITE } \\
* \text { PRESENCE * } \\
\text { PROTOTYPICALITE }\end{array}$} & PERT & 8,734 & 1 & 8,734 & 12,298 &, $001^{* * *}$ & \multirow{2}{*}{$\begin{array}{c}\text { Effets d'inter. } \\
\text { significatifs }\end{array}$} \\
\hline & NOUV & 5,440 & 1 & 5,440 & 10,372 &, $001^{* * *}$ & \\
\hline & Sucré & 1,241 & 1 & 1,241 & 2,028 & , 155 & \\
\hline & Doux & 2,409 & 1 & 2,409 & 2,073 & , 151 & \\
\hline & Intensité & 4,863 & 1 & 4,863 & 7,946 &, $005^{* * * *}$ & $\begin{array}{c}\text { Effet d'inter. } \\
\text { significatif }\end{array}$ \\
\hline \multirow{5}{*}{ Genre } & PERT & ,792 & 1 & ,792 & 1,115 & ,292 & \\
\hline & NOUV &, 216 & 1 &, 216 & ,412 &, 521 & \\
\hline & Sucré & ,015 & 1 & ,015 & ,024 & ,876 & \\
\hline & Doux & 1,446 & 1 & 1,446 & 1,244 & 265 & \\
\hline & Intensité & 137 & 1 & ,137 & ,223 & ,637 & \\
\hline \multirow{5}{*}{ Région d'origine } & PERT & 5,088 & 1 & 5,088 & 7,165 &, $008^{* * *}$ & \multirow{5}{*}{$\begin{array}{c}3 \text { Effets } \\
\text { significatifs }\end{array}$} \\
\hline & NOUV & 1,076 & 1 & 1,076 & 2,051 &, 153 & \\
\hline & Sucré & 5,612 & 1 & 5,612 & 9,168 &, $003^{* * * *}$ & \\
\hline & Doux & , 155 & 1 &, 155 & , 133 & ,715 & \\
\hline & Intensité & 4,641 & 1 & 4,641 & 7,584 &, $006^{* * * *}$ & \\
\hline \multirow{5}{*}{ Implication } & PERT & ,008 & 1 & ,008 & ,011 & ,915 & \\
\hline & NOUV &, 510 & 1 &, 510 &, 973 &, 325 & \\
\hline & Sucré & 1,530 & 1 & 1,530 & 2,499 &, 115 & \\
\hline & Doux & 1,901 & 1 & 1,901 & 1,636 & ,202 & \\
\hline & Intensité & ,227 & 1 & ,227 & ,371 & ,543 & \\
\hline
\end{tabular}




\section{FIGURES}

Figure 1. Modèle à tester

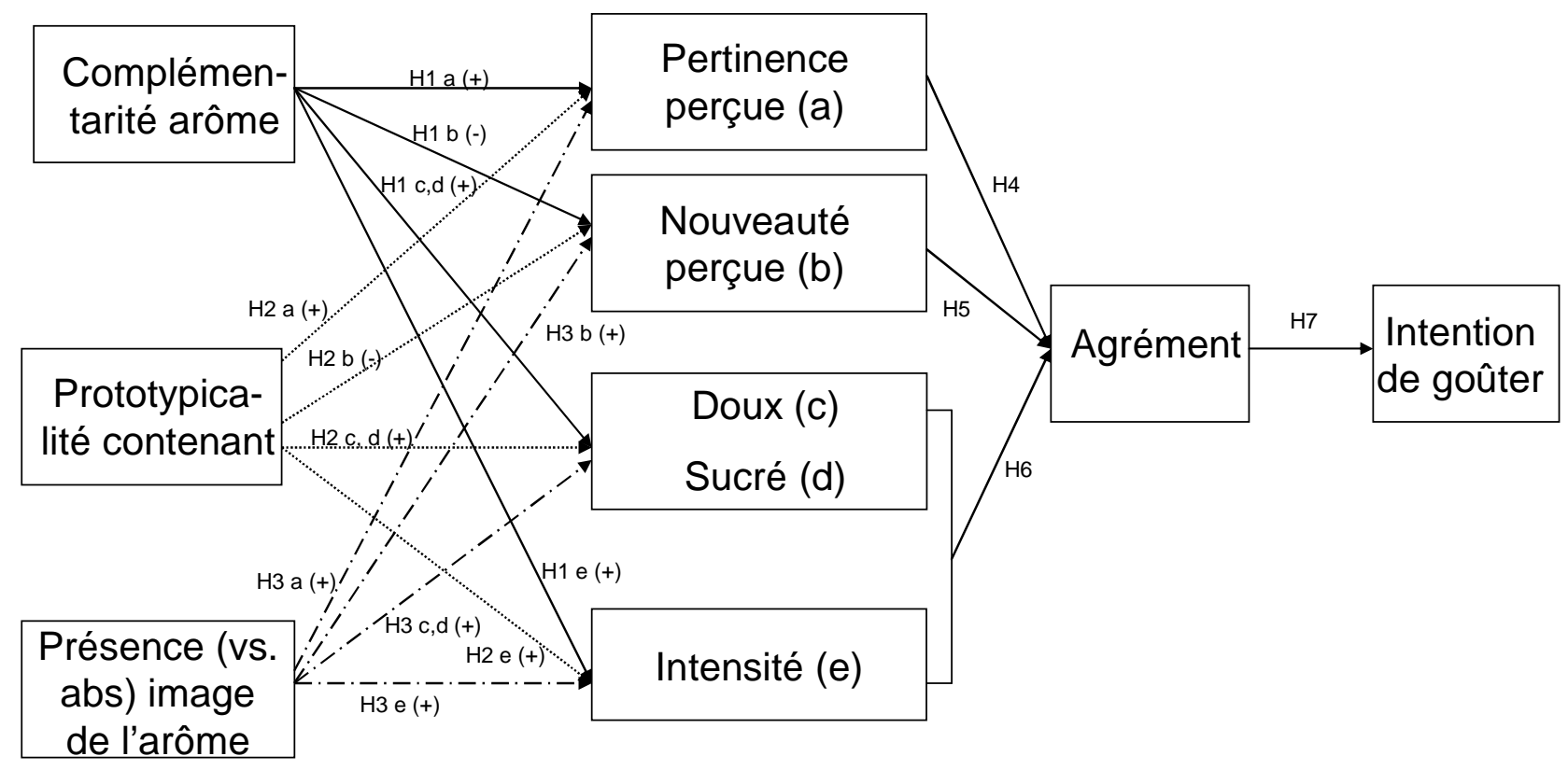

\section{Variables de contrôle}

Genre, Région d'origine, Implication 
Figure 2. Relation entre Pertinence et Agrément (Hypothèse H4)

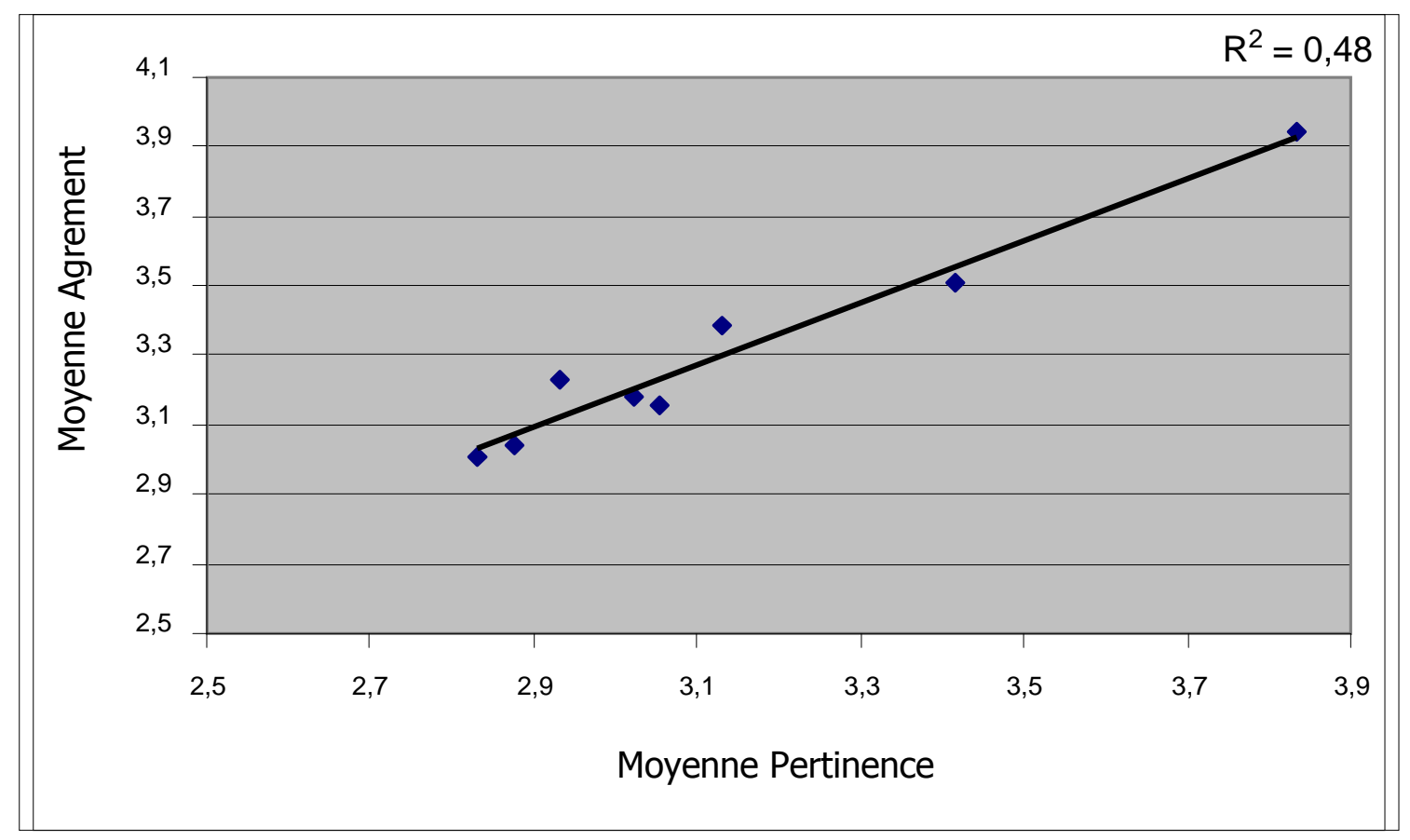


Figure 3. Lien entre Nouveauté et Agrément (hypothèse H5)

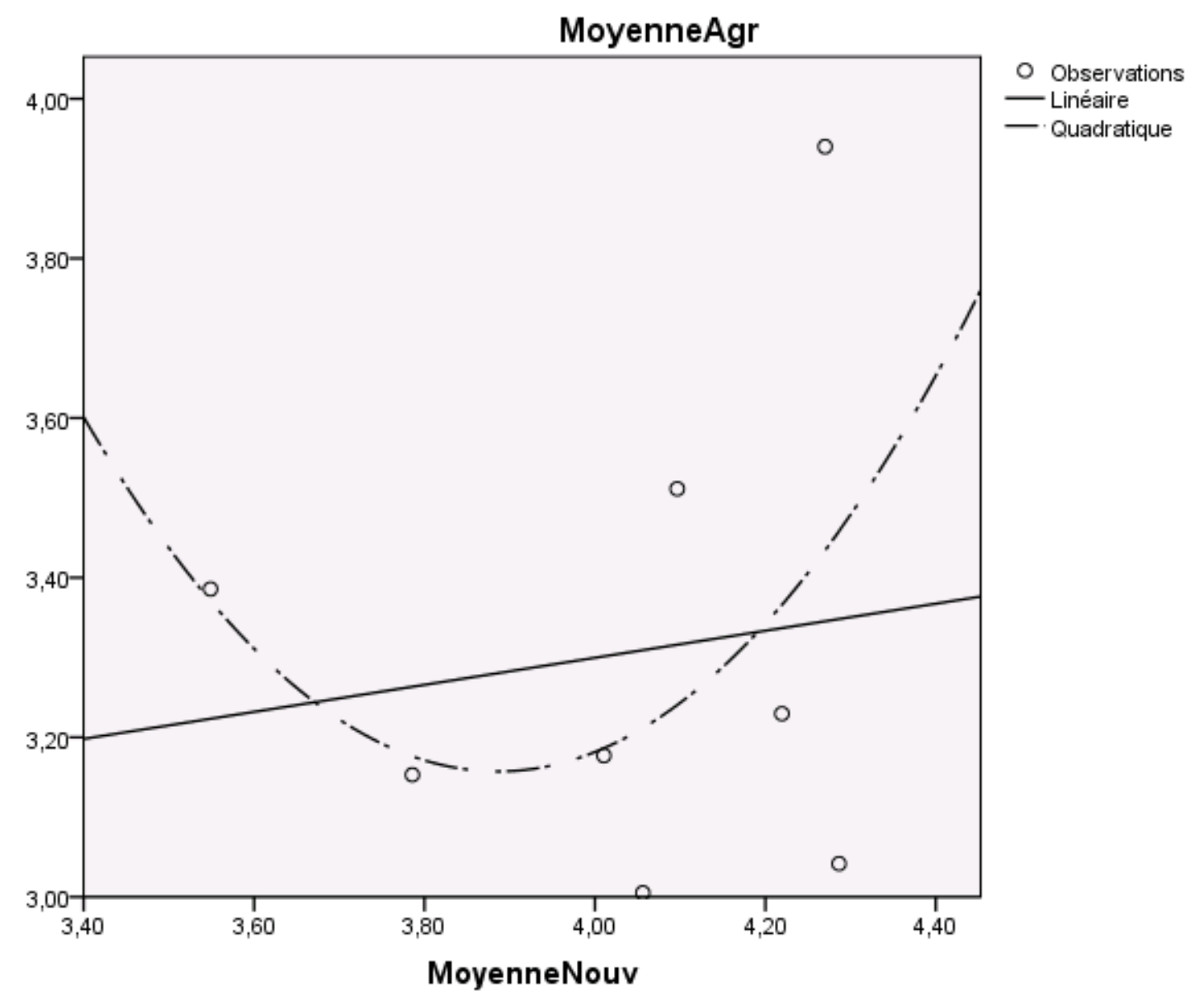




\section{BIBLIOGRAPHIE}

Aaker D.A. \& Keller K.L. (1990), Consumer Evaluations of Brand Extensions, Journal of Marketing, 54, 1, 27-41.

Alba J.W. et Hutchinson J.W. (1987), Dimensions of consumer expertise, Journal of Consumer Research, 13, 411-454.

Amine et Glerant-Glikson (2010), Catégorisation et évaluation des produits nouveaux hybrides par les consommateurs: Application à deux produits électroniques grand public, Actes du $26^{\text {ème }}$ Congrès de l'Association Française de Marketing, Le Mans - Angers, 6 et 7 juin.

Aurier P. et Fort F. (2005), Effets de la région d'origine, du produit, de la marque et de leur congruence, sur l'évaluation des consommateurs : application aux produits agro-alimentaires, Recherche et Applications en Marketing, 20, 4, 29-52.

Baron R.M. et Kenny D.A. (1986), Moderator-mediator variables distinction in social psychological research: conceptual, strategic, and statistical considerations, Journal of Personality and Social Psychology, 51, 6, 1173-1182.

Becker L., van Rompay T.J.L., Schifferstein H.N.J. et Galetzka M. (2011), Tough package, strong taste: the influence of packaging design on taste impressions and product evaluations, Food Quality and Preference, 22, 17-23.

Berkowitz M. (1987a), The influence of shape on product preferences, in Advances in Consumer Research, Vol. 14, 559.

Berkowitz M. (1987b), Product shape as a design innovation strategy, Journal of Product Innovation and Management, 4, 274-283.

Berlyne D. E. (1967), Arousal and reinforcement, in Levine D., Nebraska Symposium on Motivation. Lincoln: University of Nebraska Press.

Berlyne D.E. (1976), Psychological aesthetics, International Journal of Psychology, 11, 4355.

Bloch P.H. (1995), Seeking the ideal form: product design and consumer response, Journal of Marketing, 59 (juillet), 16-29.

Campbell M. et Keller K.L. (2003), Brand familiarity and advertising repetition effects, Journal of Consumer Research, 30 (September), 292-304. 
Celhay F. (2010), Design de packaging, appréciation esthétique et intention d'achat: l'impact du jugement d'atypicalité, Thèse de Doctorat en Sciences de Gestion, Université Bordeaux 4, Bordeaux.

Chiva M. (1992), Les aspects psychologiques des conduites alimentaires, in H. Dupin, J-L. Cuq, M-I. Malewiak, C. Leynaud-Rouaud et A-M. Berthier (coord.), Alimentation et Nutritions Humaines, Paris, ESF.

Chumpitaz Caceres Ruben et Vanhamme Joëlle (2003), Les processus modérateurs et méditeurs: distinction conceptuelle, aspects analytiques et illustrations, Recherche et Applications en Marketing, 18, 2, 67-100.

Christensen C.M. (1983), Effects of color on aroma, flavor and texture judgments of foods, Journal of Food Science, 48, 787-790.

Cohen J.B. et Basu K. (1987), Alternative models of categorization: toward a contingent processing framework, Journal of Consumer Research, 13, 4, 455-472.

Damak L. (1996), Corps du consommateur et design du produit : recherche d'une similarité ou d'une complémentarité ?, Thèse de Doctorat en Sciences de Gestion, Université ParisDauphine, Paris.

Deliza R. et Mac Fie H.J.H. (1996), The generation of sensory expectation by external cues and its effect on sensory perception and hedonic ratings : a review, Journal of Sensory Studies, 11, 2, 103-128.

Desphande R. et Hoyer W.D. (1983), Consumer decision making: strategies, cognitive efforts and perceived risk, American Marketing Association, Educator's proceedings, 88-92.

D’Hauteville F. (2003), Processus sensoriels et préférence gustative: apports de la recherche expérimentale au marketing agro-alimentaire, Revue Française de Marketing, 194, 4/5, 13-27. Edell J.A. et Staelin R. (1983), The information processing of pictures in print advertisements, Journal of Consumer Research, 10, 1, 45-61.

Fenko A., Shifferstein H.N.J. et Hekkert P. (2010), Shifts in sensory dominance between various stages of user-product interactions, Applied Ergonomics, 41, 34-40.

Fleck-Dousteyssier N. et Korchia M (2006), Les célébrités dans la publicité, le rôle de la congruence, Cahier de Recherche DMSP n 353, Université Paris Dauphine.

Fleck N. et Maille V. (2010), Trente ans de travaux contradictoires sur l'influence de la congruence perçue par le consommateur : synthèse, limites et voies de recherche, Recherche et Applications en Marketing, 25, 4, 69-92.

Gatignon H. et Xuereb J.-M. (1997), Strategic orientation of the firm and new product performance, Journal of Marketing Research, 34, 77-90. 
Garber L., Hyatt E. et Boya U (2008), Does visual package clutter obscure the communicability of food package shape?, Journal of Food Products Marketing, 14, 4, 21-32. Gielens K. et Steenkamp J-B. (2007), Drivers of acceptance of new packaged goods : an investigation across products and countries, International Journal of Research in Marketing, 24, 97-111.

Graeff T.R. et Olson J.C. (1994), Consumer inference as part of product comprehension, Advances in Consumer Research, 21, 201-207.

Heckler S.E. et Childers T.L. (1992), The role of expectancy and relevancy in memory for verbal and visual information: what is incongruency?, Journal of Consumer Research, 18, Mars, 475-492.

Hekkert P., Snelders D. et Van Wierigen P. (2003), Most advanced, yet acceptable: typicality and novelty as joint predictors of aesthetic preference in industrial design, British Journal of Psychology, 94, 111-124.

Hoegg J. et Alba J.W. (2011), Seeing is believing (Too much) : the influence of product form on perceptions of functional performance, Journal of Product and Innovation Management, 28, 346-359.

Hoyer W.D. et Brown S.P. (1990), Effect of brand awareness on choice for a common repeatpurchase product, Journal of Consumer Research, 17, 141-148.

Howell D.C. (1998), Méthodes statistiques en sciences humaines, De Boeck Université, Paris. Irmak C., Vallen B. et Sen S. (2010), You like what I like, but I don't like what you like: uniqueness motivations in product preferences, Journal of Consumer Research, 37, 3, 443455.

Jaeger S.R. et Harker F.R. (2005), Consumer evaluation of novel kiwifruit: willingness to pay, Journal of the Science of Food and Agriculture, 85, 2519-2526.

Johnson J. et Clydesdale F.M. (1982), Perceived sweetness and redness in colored sucrose solutions, Journal of Food Science, 47, 747-752.

Johnson J., Dzendolet E. et Clydesdale F.M. (1983), Psychophysical relationship between sweetness and redness in strawberry-flavored drinks, Journal of Food Protection, 46, 21-25. Johnson J., Dzendolet E., Damon R., Sawyer M et Clydesdale F.M. (1982), Psychophysical relationship between perceived sweetness and color in cherry-flavored beverages, Journal of Food Protection, 45, 601-606.

Kotler P. et Rath G.A. (1984), Design: a powerful but neglected strategic tool, Journal of Business Strategy, 5, 16-21. 
Kreuzbauer R. et Malter A.J. (2005), Embodied cognition and new product design: changing product form to influence brand categorization, Journal of Product Innovation Management, $22,165-176$.

Krider R.E., Raghubir P. et Krishna A. (2001), Pizzas: $\pi$ or square? Psychological biases in area comparisons, Marketing Science, 20, 4 (Automne), 405-425.

Ladwein R. (1995), Catégories cognitives et jugement de typicalité en comportement du consommateur, Recherche et Applications en Marketing, 10, 2, 89-100.

Ladwein R. (1998), le jugement de typicalité et le choix de la marque : le rôle de l'incertitude, Actes du $14{ }^{\text {ème }}$ Congrès International de l'Association Française de Marketing, Bordeaux.

Lavin Joseph G. \& Lawless Harry T. (1998), Effects of color and odor on judgments of sweetness among children and adults, Food Quality and Preference, 9, 4, 283-289.

Lee M. (1995), Effects of schema congruity and involvement on product evaluations, Advances in Consumer Research, 22, 210-216.

Lee Y.H. et Mason C. (1999), Responses to information incongruency in advertising : the role of expectancy, relevancy and humor, Journal of Consumer Research, 26, 2, 156-169.

Lenglet F. (2007), Comprendre le goût pour les aliments : l'impact modérateur des tendances exploratoires, Actes du XXIIIème Congrès International de l'Association Française de Marketing, 31 mai et $1^{\text {er }}$ juin, CD-ROM.

Levi-Strauss C. (1964), Le cru et le cuit, Paris, Plon.

Lichtlé M.C. (2002), Etude expérimentale de l'impact de la couleur d'une annonce publicitaire sur l'attitude envers l'annonce, Recherche et Applications en Marketing, 17, 2, 23-39.

Loken B. et Ward J. (1990), Alternative approaches to understanding the determinants of typicality, Journal of Consumer Research, 17, 111-126.

Magne S. (1999), Essai de mesure de l'attitude esthétique du consommateur envers la forme design du packaging et une variable explicative, la sensibilité esthétique personnelle, Thèse de doctorat en Sciences de Gestion, Université de Toulouse 1, Toulouse.

Maille V. (2000), La réponse du consommateur au produit parfumé: l'incidence de la congruence de l'odeur, Actes du Congrès sur les Tendances du Marketing, organisé par l'ESCP-EAP et l'Université Ca'Foscari de Venise, Venise, Novembre.

Maille V. (2007), L'intensité du goût et de la couleur de produits alimentaires : l'influence de l'incongruence perçue sur l'acceptabilité, Actes $d u 23^{\text {ème }}$ Congrès International de l'Association française de Marketing, 31 mai-1er juin, Aix-les- Bains. 
Maille Virginie et Fleck Nathalie (2011), Congruence perçue par le consommateur: vers uen clarification du concept, de sa formation et de sa mesure, Recherche et Applications en Marketing, 26, 2, 77-111.

Mandler G. (1982), The structure of value: accounting for taste, in Affect and Cognition: The 17th Annual Carnegie Symposium on Cognition, eds. Margaret S. Clarke and Susan T.

Martin I.M. et Steward D.W. (2001), The differential impact of goal congruency on attitudes, intentions, and the transfer of brand equity, Journal of Marketing Research, 38,4, 471-484.

Masson J. (2010), Effets de la modification d'un attribut constitutif d'un produit alimentaire sur son adoption par les consommateurs : le cas du vin à teneur réduite en alcool, Thèse de Doctorat en Sciences de Gestion, Université de Montpellier II.

Masterson R. (2005), The importance of creative match in television sponsorship, International Journal of Advertising, 24, 4, 505-526.

Meyers-Levy et Tybout A.M. (1989), Schema congruity as a basis for product evaluation, Journal of Consumer Research, 16, 39-54.

Mitchell A.A. et Olson J.C. (1981), Are product attribute beliefs the only mediator of advertising effects on brand attribute?, Journal of Marketing Research, 18, août, 318-332.

Nedungadi P et Hutchinson J.W. (1985), The prototypicality of brands : relationships with brand awareness, preference and usage, eds. Elizabeth C. Hirschman and Morris Holbrook, Provo, UT:Advances in Consumer Research, 12, 498-503.

Oackley M. (1990), Design and design management, in Design management: a handbook of issues and methods, M. Oackley (ed.), Cambridge, MA: Basil Blackwell Inc.

Pangborn R.M. (1960), Influence of color on the discrimination of sweetness, American Journal of Psychology, 73, 2, 229-238.

Pangborn R.M. et Hansen B. (1962), The influence of color on discrimination of sweetness and sourness in pear nectar, American Journal of Psychology, 76, 2, 315-317.

Radford S.K. et Bloch P.H. (2011), Linking Innovation to design: Consumer response to visual product newness, Journal of Product and Innovation Management, 28, 208-220.

Reed S.K. (1972), Pattern recognition and recognization, Cognitive Psychology, 3, 207-238.

Rettie, R et Brewer, C. (2000), The verbal and visual components of package design, Journal of Product and Brand Management, 9, 1, 56-70.

Rogers E.M.(1995), Diffusion of innovations, $4^{\text {th }}$ Edition, New York : The Free Press.

Rosch E. (1973), Cognitive reference points, Cognitive Psychology, 7, 532-547.

Rosch E. et Mervis C.B. (1975), Family resemblances: studies in the internal structures of categories, Cognitive Psychology, 7, 573-603. 
Rozin, P. (1976), The Selection of Foods by Rats, Humans, and Other Animals," in Advances in the Study of Behavior 6, eds. J. S. Rosenblatt, R.A. Hinde, E. Shaw, and C. Beer, 21-76.

Schifferstein, H.N.J. (2001), Effects of product beliefs on product perception and liking. In Frewer, L., Risvik, E., Schifferstein, H. Food, People and Society. A European Perspective of Consumers' Food Choices. Springer Verlag, London.

Schifferstein H.N.J., Kole A.P.W. et Mojet J. (1999), Asymmetry in the disconfirmation of expectations for natural yogurt, Appetite, 32, 307-325.

Schoormans J.P.L. et Robben H.S.J. (1997), The effect of new package on product attention, categorization and evaluation, Journal of Economic Psychology, 18, 271-287.

Sirieix L. (1999), La consommation alimentaire: problématiques, approches et voies de recherché, Recherche et Applications en Marketing, 14, 3, 1-22.

Smith R.E. , Chen J. et Yang X. (2008), The impact of advertising on the hierarchy of effects, Journal of Advertising, 37, 4, 47-61.

Stayman D.M., Alden D.L. et Smith K.H. (1992), Some effects of schematic processing on consumer expectations and disconfirmations judgments, Journal of Consumer Research, 19, 240-255.

Steenkamp J-B. et Gielens K. (2003), Consumers and Market Drivers of the trial probability of new packaged goods, Journal of Consumer Research, 30, 3, 368-384.

Strazzieri A. (1994), Mesurer l'implication durable vis-à-vis d'un produit indépendamment du risque perçu, Recherche et applications en Marketing, 9, 1, 73-91.

Tysoe M. (1985), What's wrong with Blue Potatoes?, Psychology Today, 19, 12, 6-8.

Tuorila H.M, Meiselman H.L., Cardello A.V. et Lesher L.L. (1998), Effects of expectations and the definition of product category on the acceptance of unfamiliar foods, Food quality and Preferences, 9, 6, 421-430.

Underwood, R.L., Klein N.M. et Burke R. (2001), Packaging Communication: Attentional Effects of Product Imagery, The Journal of Product and Brand Management, 10, 7, 403-422.

Underwood R.L. et Klein N.M. (2002), Packaging as brand communication: effects of products pictures on consumer responses to the package and brand, Journal of Marketing Theory and Practice, 10, 4, 58-68.

Van Rompay T.J.L., Pruyn A.T.H. et Tieke P. (2009), Symbolic meaning integration in design and its influence on product and brand evaluation, International Journal of Design, 3, $19-26$. 
Van Trijp H. C. M. et Steenkamp J-B. (1992), Consumers' variety seeking tendancy with respect to foods, measurement and managerial implications, European Review of Agricultural Economics, 19, 181-195.

Veryzer R.W. Jr (1993), The influence of unity and prototypicality on aesthetic responses to new product designs, Thèse de Doctorat, Université de Floride, Gainesville.

Veryzer R.W. Jr et Hutchinson J.W. (1998), The influence of unity and prototypicality on aesthetic responses to new product designs, Journal of Consumer Research, 24 (Mars), 374394.

Yang S. et Raghubir P. (2006), Les bouteilles peuvent-elles être transcrites en volume? L'effet de la forme de l'emballage sur la quantité à acheter, Recherche et Applications en Marketing, 21, 1, 81-100.

Zajonc R.B. et Markus H. (1982), Affective and Cognitive factors in preferences, Journal of Consumer Research, 9, 3, 123-131.

Zellner D. A. et Kautz M.A. (1990), Color affects perceived odor intensity, Journal of Experimental Psychology: human perception and performance, 16, 2, 391-397.

Zhao X., Lynch Jr. J.G. et Chen Q. (2011), Reconsidérer Baron et Kenny : mythes et vérités à propos de l'analyse de médiation, Recherche et Applications en Marketing, 26, 1, 81-95. 